\title{
Pricing FX Options in the Heston/CIR Jump-Diffusion Model with Log-Normal and Log-Uniform Jump Amplitudes
}

\author{
Rehez Ahlip ${ }^{1}$ and Ante Prodan ${ }^{2}$ \\ ${ }^{1}$ School of Computing and Mathematics, University of Western Sydney, South Penrith NSW 1797, Australia \\ ${ }^{2}$ School of Computing Engineering and Mathematics, University of Western Sydney, Sydney, NSW 1797, Australia \\ Correspondence should be addressed to Rehez Ahlip; r.ahlip@uws.edu.au
}

Received 26 November 2014; Accepted 7 May 2015

Academic Editor: Enzo Orsingher

Copyright (c) 2015 R. Ahlip and A. Prodan. This is an open access article distributed under the Creative Commons Attribution License, which permits unrestricted use, distribution, and reproduction in any medium, provided the original work is properly cited.

\begin{abstract}
We examine foreign exchange options in the jump-diffusion version of the Heston stochastic volatility model for the exchange rate with log-normal jump amplitudes and the volatility model with log-uniformly distributed jump amplitudes. We assume that the domestic and foreign stochastic interest rates are governed by the CIR dynamics. The instantaneous volatility is correlated with the dynamics of the exchange rate return, whereas the domestic and foreign short-term rates are assumed to be independent of the dynamics of the exchange rate and its volatility. The main result furnishes a semianalytical formula for the price of the foreign exchange European call option.
\end{abstract}

\section{Introduction}

We extend the results from Ahlip and Rutkowski [1] by deriving a closed-form pricing formula for the foreign exchange (FX) options in a model where the spot exchange rate and its volatility are jump-diffusions, with log-normal and loguniform jump amplitudes, respectively, whereas the domestic and foreign interest rates are governed by the Cox-IngersollRoss (CIR) dynamics postulated in [2]. In particular, our model allows for correlation between the exchange rate process and its instantaneous volatility. The interest rate processes are independent of one another, and they are also independent of the foreign exchange rate and its volatility.

In the seminal paper by Heston [3], the author noted that increasing the volatility of volatility only increases the kurtosis of spot returns and does not capture skewness. In order to capture the skewness, it is crucial to include also the properly specified correlation between the volatility and the spot exchange rate returns. In papers by Bakshi et al. [4], Bates [5], and Duffie et al. [6], the authors showed that stochastic volatility models do not offer reliable prices for close to expiration derivatives. This motivated Bates [5] and
Bakshi et al. [4] to introduce jumps to the dynamics of the exchange rate. However, as observed by Andersen and Andreasen [7] and Alizadeh et al. [8], the addition of jumps to the dynamics of the exchange rate is not sufficient to capture the sudden increase in volatility due to market turbulence. Since the overall volatility in financial markets consists of a highly persistent slow moving and rapid moving components, Eraker et al. [9] proposed to introduce jump process to the dynamics of the volatility process in order to enhance the cross-sectional impact on option prices.

More recently, D'Ippoliti et al. [10] obtained closed-form solutions, in the spirit of Heston, in a model with jumps in both spot returns of the underlying asset and its volatility. Yan and Hanson [11] consider a model in which the stock prices follow a jump-diffusion process with log-uniformly distributed jump amplitudes under the Heston volatility model. In the above-mentioned papers, the authors assume constant interest rates. Although the assumption of constant interest rates leads to highly tractable FX models, empirical results have confirmed that such models do not reflect the market reality, especially for long-dated hybrid FX products. For these products, the fluctuations of both the exchange rate 
and the interest rates are critical, so that the constant interest rates assumption is clearly inappropriate for reliable valuation and hedging.

Let us comment briefly on the existing literature in the same vein. van Haastrecht et al. [12] have extended the stochastic volatility model of Schobel and Zhu [13] to equity/currency derivatives by including stochastic interest rates and assuming all driving model factors to be instantaneously correlated. Since their model is based on the Gaussian processes, it enjoys analytical tractability even in the most general case of a full correlation structure. By contrast, when the squared volatility is driven by the CIR process and the interest rate is driven either by the Vasicek [14] or the Cox et al. [2] process, a full correlation structure leads to intractability of equity options even under a partial correlation of the driving factors, as have been documented by, among others, van Haastrecht and Pelsser [15] and Grzelak and Oosterlee $[16,17]$, who examined, in particular, the Heston/Vasicek and Heston/CIR hybrid models (see also Grzelak et al. [18], where the Schobel-Zhu/Hull-White and Heston/HullWhite models for equity derivatives are studied).

Our goal is to derive semianalytical solutions for prices of plain-vanilla FX options in a model in which the instantaneous volatility component is specified by the extended Heston model with log-normally and log-uniformly distributed jump amplitudes for the exchange rate and the volatility process, respectively, whereas the short-term interest rates for the domestic and foreign economies are governed by the independent CIR processes. The model thus incorporates important empirical characteristics of exchange rate return variability: (a) the correlation between the exchange rate and its stochastic volatility, (b) the presence of jumps in the exchange rate and volatility processes, and (c) the random character of interest rates. The practical importance of this feature of newly developed FX models is rather clear in view of the existence of complex FX products that have a long lifetime and are sensitive to smiles or skews in the market. The results obtained in this paper extend results obtained by Guoqing et al. In their model only the stock price process is subject to jumps, but the volatility of volatility is modeled by the Heston dynamics.

The paper is organized as follows. In Section 2, we set the foreign exchange model examined in this work. The options pricing problem is introduced in Section 3. The main result, Theorem 3 of Section 4, furnishes the pricing formula for FX options. It is worth stressing that the independence of volatility and interest rates appears to be a crucial assumption from the point of view of analytical tractability and thus it cannot be relaxed. Numerical illustrations of our method are provided in Section 5 where the diffusion and jump-diffusion models are compared.

\section{The Heston/CIR Jump-Diffusion Foreign Exchange Model}

Let $(\Omega, \mathscr{F}, \mathbb{P})$ be an underlying probability space. Let the exchange rate $Q=\left(Q_{t}\right)_{t \in[0, T]}$, its instantaneous squared volatility $v=\left(v_{t}\right)_{t \in[0, T]}$, the domestic short-term interest rates $r=\left(r_{t}\right)_{t \in[0, T]}$, and the foreign short-term interest rate $\widehat{r}=\left(\widehat{r}_{t}\right)_{t \in[0, T]}$ be governed by the following system of SDEs:

$$
\begin{aligned}
d Q_{t} & =\left(r_{t}-\widehat{r}_{t}-\lambda \mu_{Q}\right) Q_{t} d t+Q_{t} \sqrt{v_{t}} d W_{t}^{Q}+Q_{t-} d Z_{t}^{Q}, \\
d v_{t} & =\left(\theta-\kappa v_{t}\right) d t+\sigma_{v} \sqrt{v_{t}} d W_{t}^{v}+d Z_{t}^{v}, \\
d r_{t} & =\left(a_{d}-b_{d} r_{t}\right) d t+\sigma_{d} \sqrt{r_{t}} d W_{t}^{d}, \\
d \widehat{r}_{t} & =\left(a_{f}-b_{f} \widehat{r}_{t}\right) d t+\sigma_{f} \sqrt{\widehat{r}_{t}} d W_{t}^{f} .
\end{aligned}
$$

We work under the following standing assumptions:

(A.1) Processes $W^{\mathrm{Q}}=\left(W_{t}^{\mathrm{Q}}\right)_{t \in[0, T]}$ and $W^{v}=\left(W_{t}^{v}\right)_{t \in[0, T]}$ are correlated Brownian motions with a constant correlation coefficient, so that the quadratic covariation between the processes $W^{\mathrm{Q}}$ and $W^{v}$ satisfies $d\left[W^{\mathrm{Q}}, W^{v}\right]_{t}=\rho d t$ for some constant $\rho \in[-1,1]$.

(A.2) Processes $W^{d}=\left(W_{t}^{d}\right)_{t \in[0, T]}$ and $W^{f}=\left(W_{t}^{f}\right)_{t \in[0, T]}$ are independent Brownian motions and they are also independent of the Brownian motions $W^{\mathrm{Q}}$ and $W^{v}$; hence the processes $Q, r$, and $\widehat{r}$ are independent.

(A.3) The process $Z_{t}^{\mathrm{Q}}=\sum_{k=1}^{N_{t}^{\mathrm{Q}}} J_{k}^{\mathrm{Q}}$ is the compound Poisson process; specifically, the Poisson process $N^{\mathrm{Q}}$ has the intensity $\lambda_{Q}>0$ and the random variables $\ln \left(1+J_{k}^{\mathrm{Q}}\right)$, $k=1,2, \ldots$, have the probability distribution $N(\ln [1+$ $\left.\left.\mu_{\mathrm{Q}}\right]-(1 / 2) \sigma_{\mathrm{Q}}^{2}, \sigma_{\mathrm{Q}}^{2}\right)$; hence the jump sizes $\left(J_{k}^{\mathrm{Q}}\right)_{k=1}^{\infty}$ are $\log$-normally distributed on $(-1, \infty)$ with mean $\mu_{Q}>$ -1 .

(A.4) The process $Z_{t}^{v}=\sum_{k=1}^{N_{t}^{v}} J_{k}^{v}$ is the compound Poisson process; specifically, the Poisson process $N^{v}$ has the intensity $\lambda_{v}>0$ and the jump sizes $J_{k}^{v}$ are uniformly distributed.

(A.5) The Poisson processes $N^{Q}, N^{v}$ and sequences of random variables $\left(J_{k}^{\mathrm{Q}}\right)_{k=1}^{\infty}$ and $\left(J_{k}^{v}\right)_{k=1}^{\infty}$ are mutually independent, as well as independent of the Brownian motions $W^{\mathrm{Q}}, W^{v}, W^{d}, W^{f}$.

(A.6) The model's parameters satisfy the stability conditions: $2 \theta>\sigma_{v}^{2}>0,2 a_{d}>\sigma_{d}^{2}>0$, and $2 a_{f}>\sigma_{f}^{2}>0$ (see, e.g., Wong and Heyde [19]).

Note that we postulate that the instantaneous squared volatility process $v$, the domestic short-term interest rate $r$, and the foreign interest rate $\hat{r}$ are independent stochastic processes. We will argue in what follows that this assumption is indeed crucial for analytical tractability. For brevity, we refer to the foreign exchange model given by SDEs (1) under Assumptions (A.1)-(A.6) as the Heston/CIR jump-diffusion FX model.

\section{Foreign Exchange Call Option}

We will first establish the general representation for the value of the foreign exchange (i.e., currency) European call option with maturity $T>0$ and a constant strike level $K>0$. The probability measure $\mathbb{P}$ is interpreted as 
the domestic spot martingale measure (i.e., the domestic risk-neutral probability). We denote by $\mathbb{F}=\left(\mathscr{F}_{t}\right)_{t \in[0, T]}$ the filtration generated by the Brownian motions $W^{Q}, W^{v}, W^{d}$, $W^{f}$ and the compound Poisson processes $Z^{\mathrm{Q}}$ and $Z^{v}$. We write $\mathbb{E}_{t}^{\mathbb{P}}(\cdot)$ and $\mathbb{P}_{t}(\cdot)$ to denote the conditional expectation and the conditional probability under $\mathbb{P}$ with respect to the $\sigma$ field $\mathscr{F}_{t}$, respectively. In our computations, we will adopt the "domestic" point of view, which will frequently be represented by the subscript $d$. Similarly, we will use the subscript $f$ when referring to a foreign denominated variable. Hence the arbitrage price $C_{t}(T, K)$ of the foreign exchange call option at time $t \in[0, T]$ is given as the conditional expectation with respect to the $\sigma$-field $\mathscr{F}_{t}$ of the option's payoff at expiration discounted by the domestic money market account; that is,

$$
\begin{aligned}
C_{t}(T, K) & =\mathbb{E}_{t}^{\mathbb{P}}\left\{\exp \left(-\int_{t}^{T} r_{u} d u\right) C_{T}(T, K)\right\} \\
& =\mathbb{E}_{t}^{\mathbb{P}}\left\{\exp \left(-\int_{t}^{T} r_{u} d u\right)\left(Q_{T}-K\right)^{+}\right\},
\end{aligned}
$$

or, equivalently,

$$
\begin{aligned}
C_{t}(T, K)= & \mathbb{E}_{t}^{\mathbb{P}}\left\{\exp \left(-\int_{t}^{T} r_{u} d u\right) Q_{T} \mathbb{1}_{\left\{\mathrm{Q}_{T}>K\right\}}\right\} \\
& -K \mathbb{E}_{t}^{\mathbb{P}}\left\{\exp \left(-\int_{t}^{T} r_{u} d u\right) \mathbb{1}_{\left\{\mathrm{Q}_{T}>K\right\}}\right\} .
\end{aligned}
$$

Similarly, the arbitrage price of the domestic discount bond maturing at time $T$ equals, for every $t \in[0, T]$,

$$
B_{d}(t, T)=\mathbb{E}_{t}^{\mathbb{P}}\left\{\exp \left(-\int_{t}^{T} r_{u} d u\right)\right\},
$$

and an analogous formula holds for the price process $B_{f}(t, T)$ of the foreign discount bond under the foreign spot martingale measure (see, e.g., Chapter 14 in Musiela and Rutkowski [20]).

As a preliminary step towards the general valuation result presented in Section 4, we state the following well-known proposition (see, e.g., Cox et al. [2] or Chapter 10 in Musiela and Rutkowski [20]). It is worth stressing that we use here, in particular, the postulated independence of the foreign interest rate $\hat{r}$ and the exchange rate process $Q$. Under this standing assumption, the dynamics of the foreign bond price $B_{f}(t, T)$ under the domestic spot martingale measure $\mathbb{P}$ can be seen as an immediate consequence of formula (14.3) in Musiela and Rutkowski [20]. The simple form of the dynamics of $B_{f}(t, T)$ under $\mathbb{P}$ is a consequence of the postulated independence of $W^{f}$ and $W^{\mathrm{Q}}$ (see Assumption (A.2)). This crucial feature underpins our further calculations and thus it cannot be easily relaxed.

Proposition 1. The prices at date tof the domestic and foreign discount bonds maturing at time $T>t$ in the CIR model are given by the following expressions:

$$
\begin{aligned}
& B_{d}(t, T)=\exp \left(m_{d}(t, T)-n_{d}(t, T) r_{t}\right), \\
& B_{f}(t, T)=\exp \left(m_{f}(t, T)-n_{f}(t, T) \widehat{r}_{t}\right),
\end{aligned}
$$

where for $i \in\{d, f\}$

$$
\begin{aligned}
& m_{i}(t, T)=\frac{2 a_{i}}{\sigma_{i}^{2}} \\
& \cdot \log \left[\frac{\gamma_{i} e^{(1 / 2) b_{i}(T-t)}}{\gamma_{i} \cosh \left(\gamma_{i}(T-t)\right)+(1 / 2) b_{i} \sinh \left(\gamma_{i}(T-t)\right)}\right], \\
& n_{i}(t, T)=\frac{\sinh \left(\gamma_{i}(T-t)\right)}{\gamma_{i} \cosh \left(\gamma_{i}(T-t)\right)+(1 / 2) b_{i} \sinh \left(\gamma_{i}(T-t)\right)}, \\
& \gamma_{i}=\frac{1}{2} \sqrt{b_{i}^{2}+2 \sigma_{i}^{2}} .
\end{aligned}
$$

The dynamics of the domestic and foreign bond prices under the domestic spot martingale measure $\mathbb{P}$ are given by

$$
\begin{aligned}
& d B_{d}(t, T)=B_{d}(t, T)\left(r_{t} d t-\sigma_{d} n_{d}(t, T) \sqrt{r_{t}} d W_{t}^{d}\right), \\
& d B_{f}(t, T)=B_{f}(t, T)\left(\widehat{r}_{t} d t-\sigma_{f} n_{f}(t, T) \sqrt{\widehat{r}_{t}} d W_{t}^{f}\right) .
\end{aligned}
$$

The following result is also well known (see, e.g., Section 14.1.1 in Musiela and Rutkowski [20]).

Lemma 2. The forward exchange rate $F(t, T)$ at time $t$ for settlement date $T$ equals

$$
F(t, T)=\frac{B_{f}(t, T)}{B_{d}(t, T)} Q_{t} .
$$

Since manifestly $Q_{T}=F(T, T)$, the option's payoff at expiration can also be expressed as follows:

$$
C_{T}(T, K)=F(T, T) \mathbb{1}_{\{F(T, T)>K\}}-K \mathbb{1}_{\{F(T, T)>K\}} .
$$

Consequently, the option's value at time $t \in[0, T]$ admits the following representation:

$$
\begin{aligned}
C_{t}(T, K) & \\
= & \mathbb{E}_{t}^{\mathbb{P}}\left\{\exp \left(-\int_{t}^{T} r_{u} d u\right) F(T, T) \mathbb{1}_{\{F(T, T)>K\}}\right\} \\
& -K \mathbb{E}_{t}^{\mathbb{P}}\left\{\exp \left(-\int_{t}^{T} r_{u} d u\right) \mathbb{1}_{\{F(T, T)>K\}}\right\} .
\end{aligned}
$$

In what follows, we will frequently use the notation $x_{t}=$ $\ln F(t, T)$, where $t \in[0, T]$.

\section{Pricing Formula for the FX Call Option}

We are in a position to state the main result of the paper, which furnishes a semianalytical formula for the arbitrage price of the FX call option of European style under the Heston stochastic volatility for the exchange rate combined with the independent CIR models for the domestic and foreign short-term rates. Since the proof of Theorem 3 relies on the derivation of the conditional characteristic function of the logarithm of the exchange rate, any suitable version of the Fourier inversion technique or simulation technique can be 
applied to obtain the option price. The interested reader is referred to, for instance, Carr and Madan [21, 22] or Lord and Kahl $[23,24]$ and the references therein, as well as the recent papers by Bernard et al. [25] and Levendorskii [26] who developed and examined in detail methods with essential improvements in accuracy and/or efficiency.

Theorem 3. Let the foreign exchange model be given by SDEs (1) under Assumptions (A.1)-(A.6). Then the price of the European FX call option equals, for every $t \in[0, T]$,

$$
\begin{aligned}
C_{t}(T, K)= & Q_{t} B_{f}(t, T) P_{1}\left(t, Q_{t}, v_{t}, r_{t}, \widehat{r}_{t}, K\right) \\
& -K B_{d}(t, T) P_{2}\left(t, Q_{t}, v_{t}, r_{t}, \widehat{r}_{t}, K\right),
\end{aligned}
$$

where the bond prices $B_{d}(t, T)$ and $B_{f}(t, T)$ are given in Proposition 1 and the functions $P_{1}$ and $P_{2}$ are given $b y$, for $j=1,2$,

$$
\begin{aligned}
& P_{j}\left(t, Q_{t}, v_{t}, r_{t}, \widehat{r}_{t}, K\right) \\
& \quad=\frac{1}{2}+\frac{1}{\pi} \int_{0}^{\infty} \operatorname{Re}\left(f_{j}(\phi) \frac{\exp (-i \phi \ln K)}{i \phi}\right) d \phi,
\end{aligned}
$$

where the $\mathscr{F}_{t}$-conditional characteristic functions $f_{j}(\phi)=$ $f_{j}\left(\phi, t, Q_{t}, v_{t}, r_{t}, \widehat{r}_{t}\right), j=1,2$, of the random variable $x_{T}=$ $\ln \left(Q_{T}\right)$ under the probability measure $\widehat{\mathbb{P}}_{T}($ see Definition 8$)$ and $\mathbb{P}_{T}$ (see Definition 6), respectively, are given by

$$
\begin{aligned}
& f_{1}(\phi)=c_{t} \exp \left[\lambda_{\mathrm{Q}} \tau\left(\left(1+\mu_{\mathrm{Q}}\right)^{i \phi} e^{-(1 / 2)\left(\phi^{2}+i \phi\right) \sigma_{\mathrm{Q}}^{2}}-1\right)\right] \\
& \cdot \exp \left[-\left(i \phi \lambda_{\mathrm{Q}} \mu_{\mathrm{Q}} \tau\right.\right. \\
& +\lambda_{v} \tau\left(\frac{\sigma_{v}\left(e^{-\left(\rho(1+i \phi) / \sigma_{v}\right) a}-e^{-\left(\rho(1+i \phi) / \sigma_{v}\right) b}\right)}{\rho(1+i \phi)(b-a)}-1\right) \\
& \left.\left.+\frac{(1+i \phi) \rho}{\sigma_{v}}\left(v_{t}+\theta \tau\right)\right)\right] \exp \left[-i \phi\left(n_{d}(t, T) r_{t}\right.\right. \\
& \left.\left.+\int_{t}^{T} a_{d} n_{d}(u, T) d u\right)\right] \exp \left[( 1 + i \phi ) \left(n_{f}(t, T) \widehat{r}_{t}\right.\right. \\
& \left.\left.+\int_{t}^{T} a_{f} n_{f}(u, T) d u\right)\right] \exp \left[-G_{1}\left(\tau, s_{1}, s_{2}\right) v_{t}\right. \\
& \left.-G_{2}\left(\tau, s_{3}, s_{4}\right) r_{t}-G_{3}\left(\tau, s_{5}, s_{6}\right) \widehat{r}_{t}\right] \\
& \cdot \exp \left[-\theta H_{1}\left(\tau, s_{1}, s_{2}\right)-a_{d} H_{2}\left(\tau, s_{3}, s_{4}\right)\right. \\
& \left.+a_{f} H_{3}\left(\tau, s_{5}, s_{6}\right)\right]
\end{aligned}
$$

$$
\begin{aligned}
& f_{2}(\phi)=c_{t} \exp \left[\lambda_{\mathrm{Q}} \tau\left(\left(1+\mu_{\mathrm{Q}}\right)^{i \phi} e^{-(1 / 2)\left(\phi^{2}+i \phi\right) \sigma_{\mathrm{Q}}^{2}}-1\right)\right] \\
& \cdot \exp \left[-\left(i \phi \lambda_{\mathrm{Q}} \mu_{\mathrm{Q}} \tau\right.\right. \\
& +\lambda_{v} \tau\left(\frac{\sigma_{v}\left(e^{-\left(\rho(i \phi) / \sigma_{v}\right) a}-e^{-\left(\rho(i \phi) / \sigma_{v}\right) b}\right)}{\rho(i \phi)(b-a)}-1\right) \\
& \left.\left.+\frac{i \phi \rho}{\sigma_{v}}\left(v_{t}+\theta \tau\right)\right)\right] \exp \left[( 1 - i \phi ) \left(n_{d}(t, T) r_{t}\right.\right. \\
& \left.\left.+\int_{t}^{T} a_{d} n_{d}(u, T) d u\right)\right] \exp \left[i \phi \left(n_{f}(t, T) \widehat{r}_{t}\right.\right. \\
& \left.\left.+\int_{t}^{T} a_{f} n_{f}(u, T) d u\right)\right] \exp \left[-G_{1}\left(\tau, q_{1}, q_{2}\right) v_{t}\right. \\
& \left.-G_{2}\left(\tau, q_{3}, q_{4}\right) r_{t}-G_{3}\left(\tau, q_{5}, q_{6}\right)\right] \\
& +\exp \left[-\theta H_{1}\left(\tau, q_{1}, q_{2}\right)-a_{d} H_{2}\left(\tau, q_{3}, q_{4}\right)\right. \\
& \left.\quad-a_{f} H_{3}\left(\tau, q_{5}, q_{6}\right)\right]
\end{aligned}
$$

where the functions $G_{1}, G_{2}, G_{3}, H_{1}, H_{2}, H_{3}$ are given in Lemma 5 and $c_{t}$ equals

$$
c_{t}=\exp \left(i \phi x_{t}\right)=\exp (i \phi \ln F(t, T)) .
$$

Moreover, the constants $s_{1}, s_{2}, s_{3}, s_{4}, s_{5}, s_{6}$ are given by

$$
\begin{aligned}
& s_{1}=-\frac{(1+i \phi) \rho}{\sigma_{v}}, \\
& s_{2}=-\frac{(1+i \phi)^{2}\left(1-\rho^{2}\right)}{2}-\frac{(1+i \phi) \rho \kappa}{\sigma_{v}}+\frac{1+i \phi}{2}, \\
& s_{3}=0, \\
& s_{4}=-i \phi, \\
& s_{5}=0, \\
& s_{6}=1+i \phi,
\end{aligned}
$$

and the constants $q_{1}, q_{2}, q_{3}, q_{4}, q_{5}, q_{6}$ equal

$$
\begin{aligned}
& q_{1}=-\frac{i \phi \rho}{\sigma_{v}}, \\
& q_{2}=-\frac{(i \phi)^{2}\left(1-\rho^{2}\right)}{2}-\frac{i \phi \rho \kappa}{\sigma_{v}}+\frac{i \phi}{2}, \\
& q_{3}=0 \\
& q_{4}=1-i \phi \\
& q_{5}=0 \\
& q_{6}=i \phi .
\end{aligned}
$$


4.1. Auxiliary Results. The proof of Theorem 3 hinges on a number of lemmas. We start by stating the well-known result, which can be easily obtained from Proposition 8.6.3.4 in Jeanblanc et al. [27]. Let us denote $\tau=T-t$ and let us set, for all $0 \leq t<T$,

$$
J^{\mathrm{Q}}(t, T):=\sum_{k=N_{t}^{\mathrm{Q}}+1}^{N_{T}^{\mathrm{Q}}} \ln \left(1+J_{k}^{\mathrm{Q}}\right) .
$$

Note that we use here Assumptions (A.3)-(A.5). The property (A.3) (resp., (A.4)) implies that the random variable $J^{\mathrm{Q}}(t, T)$ (resp., $Z_{T}^{v}-Z_{t}^{v}$ ) is independent of the $\sigma$-field $\mathscr{F}_{t}$. Let $\nu_{1}$ stand for the Gaussian distribution $N\left(\ln \left(1+\mu_{\mathrm{Q}}\right)-(1 / 2) \sigma_{Q}^{2}, \sigma_{Q}^{2}\right)$ and let $v_{2}$ stand for the uniform distribution with density:

$$
v_{2}(z)=\frac{1}{b-a} \begin{cases}1, & a<z<b, \\ 0, & \text { else, }\end{cases}
$$

where $0<a<b$.

Lemma 4. (i) Under Assumptions (A.3) and (A.5), the following equalities are valid:

$$
\begin{aligned}
\mathbb{E}_{t}^{\mathbb{P}} & \left\{\exp \left(i \phi J^{\mathrm{Q}}(t, T)\right)\right\} \\
& =\mathbb{E}_{t}^{\mathbb{P}}\left\{\exp \left(i \phi \sum_{k=N_{t}^{\mathrm{Q}}+1}^{N_{T}^{\mathrm{Q}}} \ln \left(1+J_{k}^{\mathrm{Q}}\right)\right)\right\} \\
& =\exp \left[\lambda_{\mathrm{Q}} \tau \int_{-\infty}^{+\infty}\left(e^{i \phi z}-1\right) \nu_{1}(d z)\right] \\
& =\exp \left[\lambda_{\mathrm{Q}} \tau\left(\left(1+\mu_{\mathrm{Q}}\right)^{i \phi} e^{-(1 / 2) \sigma_{\mathrm{Q}}^{2}\left(\phi^{2}+i \phi\right)}-1\right)\right] .
\end{aligned}
$$

(ii) Under Assumptions (A.4) and (A.5), the following equalities are valid for $c=a+b i$ with $a \leq 0$ :

$$
\begin{aligned}
\mathbb{E}_{t}^{\mathbb{P}} & \left\{\exp \left(c\left(Z_{T}^{v}-Z_{t}^{v}\right)\right)\right\}=\mathbb{E}_{t}^{\mathbb{P}}\left\{\exp \left(c \sum_{k=N_{t}^{v}+1}^{N_{T}^{v}} J_{k}^{v}\right)\right\} \\
& =\exp \left[\lambda_{v} \tau \int_{-\infty}^{+\infty}\left(e^{c z}-1\right) \nu_{2}(d z)\right] \\
& =\exp \left[\lambda_{v} \tau\left(\frac{e^{c b}-e^{c a}}{c(b-a)}-1\right)\right] .
\end{aligned}
$$

The next result extends Lemma 6.1 in Ahlip and Rutkowski [28] (see also Duffie et al. [6]) where the model without the jump component in the dynamics of $v$ was examined.

Lemma 5. Let the dynamics of processes $v, r$, and $\hat{r}$ be given by SDEs (1) with independent Brownian motions $W^{v}, W^{d}$, and $W^{f}$. For any complex numbers $\mu, \lambda, \tilde{\mu}, \tilde{\lambda}, \widehat{\mu}, \hat{\lambda}$, we set

$$
\begin{gathered}
F\left(\tau, v_{t}, r_{t}, \widehat{r}_{t}\right)=\mathbb{E}_{t}^{\mathbb{P}}\left\{\operatorname { e x p } \left(-\lambda v_{T}-\mu \int_{t}^{T} v_{u} d u-\tilde{\lambda} r_{T}\right.\right. \\
\left.\left.-\tilde{\mu} \int_{t}^{T} r_{u} d u-\hat{\lambda} \widehat{r}_{T}-\widehat{\mu} \int_{t}^{T} \widehat{r}_{u} d u\right)\right\} .
\end{gathered}
$$

Then

$$
\begin{aligned}
& F\left(\tau, v_{t}, r_{t}, \widehat{r}_{t}\right)=\exp \left[-G_{1}(\tau, \lambda, \mu) v_{t}-G_{2}(\tau, \tilde{\lambda}, \tilde{\mu}) r_{t}\right. \\
& -G_{3}(\tau, \hat{\lambda}, \widehat{\mu}) \widehat{r}_{t}-\theta H_{1}(\tau, \lambda, \mu)-a_{d} H_{2}(\tau, \tilde{\lambda}, \tilde{\mu}) \\
& \left.-a_{f} H_{3}(\tau, \widehat{\lambda}, \widehat{\mu})\right],
\end{aligned}
$$

where

$$
\begin{aligned}
& G_{1}(\tau, \lambda, \mu) \\
& =\frac{\lambda\left[(\gamma+\kappa)+e^{\gamma \tau}(\gamma-\kappa)\right]+2 \mu\left(e^{\gamma \tau}-1\right)}{\sigma_{v}^{2} \lambda\left(e^{\gamma \tau}-1\right)+\gamma-\kappa+e^{\gamma \tau}(\gamma+\kappa)}, \\
& G_{2}(\tau, \tilde{\lambda}, \tilde{\mu}) \\
& =\frac{\tilde{\lambda}\left[\left(\tilde{\gamma}+b_{d}\right)+e^{\tilde{\gamma} \tau}\left(\tilde{\gamma}-b_{d}\right)\right]+2 \tilde{\mu}\left(e^{\tilde{\gamma} \tau}-1\right)}{\sigma_{d}^{2} \tilde{\lambda}\left(e^{\tilde{\gamma} \tau}-1\right)+\tilde{\gamma}-b_{d}+e^{\tilde{\gamma} \tau}\left(\tilde{\gamma}+b_{d}\right)}, \\
& G_{3}(\tau, \widehat{\lambda}, \widehat{\mu}) \\
& =\frac{\hat{\lambda}\left[\left(\widehat{\gamma}+b_{f}\right)+e^{\hat{\gamma} \tau}\left(\widehat{\gamma}-b_{f}\right)\right]+2 \widehat{\mu}\left(e^{\widehat{\gamma} \tau}-1\right)}{\sigma_{f}^{2} \hat{\lambda}\left(e^{\hat{\gamma} \tau}-1\right)+\widehat{\gamma}-b_{f}+e^{\widehat{\gamma} \tau}\left(\hat{\gamma}+b_{f}\right)}, \\
& H_{1}(\tau, \lambda, \mu)=\int_{0}^{\tau}\left(G_{1}(t, \lambda, \mu)\right. \\
& \left.+\frac{\lambda_{v}}{\theta}\left(1+\frac{e^{-b G_{1}(t, \lambda, \mu)}-e^{-a G_{1}(t, \lambda, \mu)}}{G_{1}(t, \lambda, \mu)(b-a)}\right)\right) d t, \\
& H_{2}(\tau, \tilde{\lambda}, \tilde{\mu})=-\frac{2}{\sigma_{d}^{2}} \\
& \cdot \ln \left(\frac{2 \widetilde{\gamma} e^{\left(\tilde{\gamma}+b_{d}\right) \tau / 2}}{\sigma_{d}^{2} \tilde{\lambda}\left(e^{\tilde{\gamma} \tau}-1\right)+\tilde{\gamma}-b_{d}+e^{\tilde{\gamma} \tau}\left(\tilde{\gamma}+b_{d}\right)}\right), \\
& H_{3}(\tau, \hat{\lambda}, \widehat{\mu})=-\frac{2}{\sigma_{f}^{2}} \\
& \cdot \ln \left(\frac{2 \widehat{\gamma} e^{\left(\hat{\gamma}+b_{f}\right) \tau / 2}}{\sigma_{f}^{2} \hat{\lambda}\left(e^{\widehat{\gamma} \tau}-1\right)+\widehat{\gamma}-b_{f}+e^{\hat{\gamma} \tau}\left(\widehat{\gamma}+b_{f}\right)}\right),
\end{aligned}
$$

where one denotes $\gamma=\sqrt{\kappa^{2}+2 \sigma_{v}^{2} \mu}, \widetilde{\gamma}=\sqrt{b_{d}^{2}+2 \sigma_{d}^{2} \widetilde{\mu}}$, and $\widehat{\gamma}=$ $\sqrt{b_{f}^{2}+2 \sigma_{f}^{2} \widehat{\mu}}$

Proof. For the reader's convenience, we sketch the proof of the lemma. Let us set, for $t \in[0, T]$,

$$
\begin{aligned}
M_{t} & =F\left(\tau, v_{t}, r_{t}, \widehat{r}_{t}\right) \\
& \cdot \exp \left(-\mu \int_{0}^{t} v_{u} d u-\tilde{\mu} \int_{0}^{t} r_{u} d u-\widehat{\mu} \int_{0}^{t} \widehat{r}_{u} d u\right) .
\end{aligned}
$$


Then the process $M=\left(M_{t}\right)_{t \in[0, T]}$ satisfies

$$
\begin{aligned}
M_{t} & =\mathbb{E}_{t}^{\mathbb{P}}\left\{\operatorname { e x p } \left(-\lambda v_{T}-\mu \int_{0}^{T} v_{u} d u-\tilde{\lambda} r_{T}\right.\right. \\
& \left.\left.-\tilde{\mu} \int_{0}^{T} r_{u} d u-\widehat{\lambda} \widehat{r}_{T}-\widehat{\mu} \int_{0}^{T} r_{u} d u\right)\right\},
\end{aligned}
$$

and thus it is an $\mathbb{F}$-martingale under $\mathbb{P}$. By applying the Itô formula to the right-hand side in (25) and by setting the drift term in the dynamics of $M$ to be zero, we deduce that the function $F(\tau, v, r, \widehat{r})$ satisfies the following partial integrodifferential equation (PIDE):

$$
\begin{aligned}
-\frac{\partial F}{\partial \tau} & +\frac{1}{2} \sigma_{v}^{2} v \frac{\partial^{2} F}{\partial v^{2}} \\
& +\lambda_{v} \int_{0}^{\infty}(F(\tau, v+z, r, \widehat{r})-F(\tau, v, r, \widehat{r})) \nu_{2}(d z) \\
& +\frac{1}{2} \sigma_{d}^{2} r \frac{\partial^{2} F}{\partial r^{2}}+\frac{1}{2} \sigma_{f}^{2} \widehat{r} \frac{\partial^{2} F}{\partial \widehat{r}^{2}}+(\theta-\kappa v) \frac{\partial F}{\partial v} \\
& +\left(a_{d}-b_{d} r\right) \frac{\partial F}{\partial r}+\left(a_{f}-b_{f} \widehat{r}\right) \frac{\partial F}{\partial \widehat{r}} \\
& -(\mu v+\widetilde{\mu} r+\widehat{\mu} \widehat{r}) F=0,
\end{aligned}
$$

with the initial condition $F(0, v, r, \widehat{r})=\exp (-\lambda v-\tilde{\lambda} r-\widehat{\lambda} \widehat{r})$. We search for a solution to this PIDE in the form

$$
\begin{aligned}
& F(\tau, v, r, \widehat{r})=\exp \left[-G_{1}(\tau, \lambda, \mu) v-G_{2}(\tau, \tilde{\lambda}, \tilde{\mu}) r\right. \\
& \quad-G_{3}(\tau, \widehat{\lambda}, \widehat{\mu}) \hat{r}-\theta H_{1}(\tau, \lambda, \mu)-a_{d} H_{2}(\tau, \tilde{\lambda}, \tilde{\mu}) \\
& \left.\quad-a_{f} H_{3}(\tau, \widehat{\lambda}, \widehat{\mu})\right],
\end{aligned}
$$

with

$$
\begin{aligned}
& G_{1}(0, \lambda, \mu)=\lambda, \\
& G_{2}(0, \tilde{\lambda}, \tilde{\mu})=\tilde{\lambda}, \\
& G_{3}(0, \hat{\lambda}, \widehat{\mu})=\hat{\lambda}, \\
& H_{1}(0, \lambda, \mu)=H_{2}(0, \tilde{\lambda}, \tilde{\mu})=H_{3}(0, \hat{\lambda}, \widehat{\mu})=0 .
\end{aligned}
$$

By substituting this expression in the PIDE and using part (ii) in Lemma 4, we obtain the following system of ODEs for the functions $G_{1}, G_{2}, G_{3}, H_{1}, H_{2}, H_{3}$ (for brevity, we suppress the last three arguments):

$$
\begin{aligned}
& \frac{\partial G_{1}(\tau)}{\partial \tau}=-\frac{1}{2} \sigma_{v}^{2} G_{1}^{2}(\tau)-\kappa G_{1}(\tau)+\mu \\
& \frac{\partial H_{1}(\tau)}{\partial \tau}=G_{1}(\tau)+\frac{\lambda_{v}}{\theta}\left(1+\frac{e^{-b G_{1}(\tau)}-e^{-a G_{1}(\tau)}}{G_{1}(\tau)(b-a)}\right), \\
& \frac{\partial G_{2}(\tau)}{\partial \tau}=-\frac{1}{2} \sigma_{d}^{2} G_{2}^{2}(\tau)-b_{d} G_{2}(\tau)+\tilde{\mu} \\
& \frac{\partial H_{2}(\tau)}{\partial \tau}=G_{2}(\tau) \\
& \frac{\partial G_{3}(\tau)}{\partial \tau}=-\frac{1}{2} \sigma_{f}^{2} G_{3}^{2}(\tau)-b_{f} G_{3}(\tau)+\widehat{\mu} \\
& \frac{\partial H_{3}(\tau)}{\partial \tau}=G_{3}(\tau) .
\end{aligned}
$$

By solving these equations, we obtain the stated formulae.

Under the assumptions of Lemma 5, it is possible to factorize $F$ as a product of two conditional expectations. This means that the functions $G_{1}\left(H_{1}\right), G_{2}\left(H_{2}\right)$, and $G_{3}\left(H_{3}\right)$ are of the same form, except that they correspond to different sets of parameters, $\theta, \kappa, \sigma_{v}$ for $G_{1}, H_{1}, a_{d}, b_{d}, \sigma_{r}$ for $G_{2}, H_{2}$, and $a_{f}$, $b_{f}, \sigma_{f}$ for $G_{3}, H_{3}$. Note, however, that the roles played by the processes $v, r$, and $\widehat{r}$ in our model are clearly different.

It should also be stressed that no closed-form analytical expression for $F\left(\tau, v_{t}, r_{t}, \widehat{r}_{t}\right)$ is available in the case of correlated Brownian motions $W^{v}, W^{r}, W^{f}$. Brigo and Alfonsi [29], who deal with this issue in a different context, propose to use a simple Gaussian approximation, instead of the exact solution. More recently, Grzelak and Oosterlee [16] proposed more sophisticated approximations in the framework of the Heston/CIR hybrid model. We do not follow this path here, however, and we focus instead on finding a semianalytical solution, since this goal can be achieved under Assumptions (A.1)-(A.6).

Let us now introduce a convenient change of the underlying probability measure, from the domestic spot martingale measure $\mathbb{P}$ to the domestic forward martingale measure $\mathbb{P}_{T}$.

Definition 6. The domestic forward martingale measure $\mathbb{P}_{T}$, equivalent to $\mathbb{P}$ on $\left(\Omega, \mathscr{F}_{T}\right)$, is defined by the RadonNikodým derivative process $\eta=\left(\eta_{t}\right)_{t \in[0, T]}$, where

$$
\begin{aligned}
\eta_{t} & =\left.\frac{d \mathbb{P}_{T}}{d \mathbb{P}}\right|_{\mathscr{F}_{t}}=\exp \left(-\int_{0}^{t} \sigma_{d} n_{d}(u, T) \sqrt{r_{u}} d W_{u}^{d}\right. \\
& \left.-\frac{1}{2} \int_{0}^{t} \sigma_{d}^{2} n_{d}^{2}(u, T) r_{u} d u\right) .
\end{aligned}
$$

An application of the Girsanov theorem shows that the process $W^{T}=\left(W_{t}^{T}\right)_{t \in[0, T]}$, which is given by the equality

$$
W_{t}^{T}=W_{t}^{d}+\int_{0}^{t} \sigma_{d} n_{d}(u, T) \sqrt{r_{u}} d u,
$$


is the Brownian motion under the domestic forward martingale measure $\mathbb{P}_{T}$. Using the standard change of a numéraire technique, one can check that the price of the European foreign exchange call option admits the following representation under the probability measure $\mathbb{P}_{T}$ :

$$
\begin{aligned}
C_{t}(T, K)= & B_{d}(t, T) \mathbb{E}_{t}^{\mathbb{P}_{T}}\left(F(T, T) \mathbb{1}_{\{F(T, T)>K\}}\right) \\
& -K B_{d}(t, T) \mathbb{E}_{t}^{\mathbb{P}_{T}}\left(\mathbb{1}_{\{F(T, T)>K\}}\right) .
\end{aligned}
$$

The following auxiliary result is easy to establish and thus its proof is omitted. Recall that $J^{\mathrm{Q}}(t, T)$ is given by equality (18).

Lemma 7. Under Assumptions (A.1)-(A.6), the dynamics of the forward exchange rate $F(t, T)$ under the domestic forward martingale measure $\mathbb{P}_{T}$ are given by the $S D E$ :

$$
\begin{aligned}
& d F(t, T)=F(t, T)\left(d Z_{t}^{\mathrm{Q}}-\lambda_{\mathrm{Q}} \mu_{\mathrm{Q}} d t+\sqrt{v_{t}} d W_{t}^{\mathrm{Q}}\right. \\
& \left.\quad+\sigma_{d} n_{d}(t, T) \sqrt{r_{t}} d W_{t}^{T}-\sigma_{f} n_{f}(t, T) \sqrt{\widehat{r}_{t}} d W_{t}^{f}\right),
\end{aligned}
$$

or, equivalently,

$$
\begin{gathered}
F(T, T)=F(t, T) \exp \left(J^{\mathrm{Q}}(t, T)-\lambda_{\mathrm{Q}} \mu_{\mathrm{Q}}(T-t)\right. \\
\left.+\int_{t}^{T} \widetilde{\sigma}_{F}(u, T) \cdot d \widetilde{W}_{u}^{T}-\frac{1}{2} \int_{t}^{T}\left\|\widetilde{\sigma}_{F}(u, T)\right\|^{2} d u\right),
\end{gathered}
$$

where the dot $\cdot$ denotes the inner product in $\mathbb{R}^{3},\left(\widetilde{\sigma}_{F}(t, T)\right)_{t \in[0, T}$ is the $\mathbb{R}^{3}$-valued process (row vector) given by

$$
\widetilde{\sigma}_{F}(t, T)=\left[\sqrt{v_{t}}, \sigma_{d} n_{d}(t, T) \sqrt{r_{t}},-\sigma_{f} n_{f}(t, T) \sqrt{\widehat{r}_{t}}\right],
$$

and $\widetilde{W}^{T}=\left(\widetilde{W}_{t}^{T}\right)_{t \in[0, T]}$ stands for the $\mathbb{R}^{3}$-valued process (column vector) given by

$$
\widetilde{W}_{t}^{T}=\left[W_{t}^{Q}, W_{t}^{T}, W_{t}^{f}\right]^{*}
$$

It is easy to check that, under Assumptions (A.1)-(A.6), the process $\widetilde{W}^{T}$ is the three-dimensional standard Brownian motion under $\mathbb{P}_{T}$. In view of Lemma 7 , we have that

$$
\begin{gathered}
B_{d}(t, T) \mathbb{E}_{t}^{\mathbb{P}_{T}}\left(F(T, T) \mathbb{1}_{\{F(T, T)>K\}}\right)=B_{d}(t, T) \\
\cdot \mathbb{E}_{t}^{\mathbb{P}_{T}}\left\{F ( t , T ) \operatorname { e x p } \left(J^{\mathrm{Q}}(t, T)-\lambda_{\mathrm{Q}} \mu_{\mathrm{Q}}(T-t)\right.\right. \\
\left.+\int_{t}^{T} \widetilde{\sigma}_{F}(u, T) \cdot d \widetilde{W}_{u}^{T}-\frac{1}{2} \int_{t}^{T}\left\|\widetilde{\sigma}_{F}(u, T)\right\|^{2} d u\right) \\
\left.\cdot \mathbb{1}_{\{F(T, T)>K\}}\right\}=Q_{t} B_{f}(t, T) \mathbb{E}_{t}^{\mathbb{P}_{T}}\left\{\operatorname { e x p } \left(J^{\mathrm{Q}}(t, T)\right.\right. \\
-\lambda_{\mathrm{Q}} \mu_{\mathrm{Q}}(T-t)+\int_{t}^{T} \widetilde{\sigma}_{F}(u, T) \cdot d \widetilde{W}_{u}^{T} \\
\left.\left.-\frac{1}{2} \int_{t}^{T}\left\|\widetilde{\sigma}_{F}(u, T)\right\|^{2} d u\right) \mathbb{1}_{\{F(T, T)>K\}}\right\} .
\end{gathered}
$$

To deal with the first term in the right-hand side of (33), we introduce another auxiliary probability measure.

Definition 8. The modified domestic forward martingale measure $\widehat{\mathbb{P}}_{T}$, equivalent to $\mathbb{P}_{T}$ on $\left(\Omega, \mathscr{F}_{T}\right)$, is defined by the Radon-Nikodým derivative process $\widehat{\eta}=\left(\widehat{\eta}_{t}\right)_{t \in[0, T]}$, where

$$
\begin{aligned}
\widehat{\eta}_{t} & =\left.\frac{d \widehat{\mathbb{P}}_{T}}{d \mathbb{P}_{T}}\right|_{\widetilde{F}_{t}} \\
& =\exp \left(\int_{0}^{t} \widetilde{\sigma}_{F}(u, T) \cdot d \widetilde{W}_{u}^{T}-\frac{1}{2} \int_{0}^{t}\left\|\widetilde{\sigma}_{F}(u, T)\right\|^{2} d u\right) .
\end{aligned}
$$

Using Lemma 7 and (8), we obtain

$$
\begin{aligned}
B_{d}(t, T) \mathbb{E}_{t}^{\mathbb{P}_{T}}\left(F(T, T) \mathbb{1}_{\{F(T, T)>K\}}\right) \\
=Q_{t} B_{f}(t, T) \frac{\mathbb{E}_{t}^{\mathbb{P}_{T}}\left(\mathbb{1}_{\{F(T, T)>K\}} \widehat{\eta}_{T}\right)}{\mathbb{E}_{t}^{\mathbb{P}_{T}\left(\hat{\eta}_{T}\right)}}
\end{aligned}
$$

and thus the Bayes formula and Definition 8 yield

$$
\begin{array}{r}
B_{d}(t, T) \mathbb{E}_{\mathrm{t}}^{\mathbb{P}_{T}}\left(F(T, T) \mathbb{1}_{\{F(T, T)>K\}}\right) \\
=Q_{t} B_{f}(t, T) \mathbb{E}_{t}^{\widehat{\mathbb{P}}_{T}}\left(\mathbb{1}_{\{F(T, T)>K\}}\right) .
\end{array}
$$

This shows that $\widehat{\mathbb{P}}_{T}$ is a martingale measure associated with the choice of the price process $Q_{t} B_{f}(t, T)$ as a numéraire asset. We are now in a position to state the following lemma.

Lemma 9. The price of the FX call option satisfies

$$
\begin{aligned}
C_{t}(T, K)= & Q_{t} B_{f}(t, T) \widehat{\mathbb{P}}_{T}\left(Q_{T}>K \mid \mathscr{F}_{t}\right) \\
& -K B_{d}(t, T) \mathbb{P}_{T}\left(Q_{T}>K \mid \mathscr{F}_{t}\right),
\end{aligned}
$$

or, equivalently,

$$
\begin{aligned}
C_{t}(T, K)= & Q_{t} B_{f}(t, T) \widehat{\mathbb{P}}_{T}\left(x_{T}>\ln K \mid \mathscr{F}_{t}\right) \\
& -K B_{d}(t, T) \mathbb{P}_{T}\left(x_{T}>\ln K \mid \mathscr{F}_{t}\right) .
\end{aligned}
$$

To complete the proof Theorem 3, it remains to evaluate the conditional probabilities arising in formula (43). By another application of the Girsanov theorem, one can check that the process $(Q, v, r, \widehat{r})$ has the Markov property under the probability measures $\mathbb{P}_{T}$ and $\widehat{\mathbb{P}}_{T}$. In view of Proposition 1 and Lemma 2 , the random variable $x_{T}$ is a function of $Q_{T}, r_{T}$, and $\widehat{r}_{T}$. We thus conclude that

$$
\begin{aligned}
C_{t}(T, K)= & Q_{t} B_{f}(t, T) P_{1}\left(t, Q_{t}, v_{t}, r_{t}, \widehat{r}_{t}, K\right) \\
& -K B_{d}(t, T) P_{2}\left(t, Q_{t}, v_{t}, r_{t}, \widehat{r}_{t}, K\right),
\end{aligned}
$$


where we denote

$$
\begin{aligned}
& P_{1}\left(t, Q_{t}, v_{t}, r_{t}, \widehat{r}_{t}, K\right)=\widehat{\mathbb{P}}_{T}\left(x_{T}>\ln K \mid Q_{t}, v_{t}, r_{t}, \widehat{r}_{t}\right), \\
& P_{2}\left(t, Q_{t}, v_{t}, r_{t}, \widehat{r}_{t}, K\right)=\mathbb{P}_{T}\left(x_{T}>\ln K \mid Q_{t}, v_{t}, r_{t}, \widehat{r}_{t}\right) .
\end{aligned}
$$

To obtain explicit formulae for the conditional probabilities above, it suffices to derive the corresponding conditional characteristic functions:

$$
\begin{aligned}
& f_{1}\left(\phi, t, Q_{t}, v_{t}, r_{t}, \widehat{r}_{t}\right)=\mathbb{E}_{t}^{\widehat{\mathbb{P}}_{T}}\left[\exp \left(i \phi x_{T}\right)\right], \\
& f_{2}\left(\phi, t, Q_{t}, v_{t}, r_{t}, \widehat{r}_{t}\right)=\mathbb{E}_{t}^{\mathbb{P}_{T}}\left[\exp \left(i \phi x_{T}\right)\right] .
\end{aligned}
$$

The idea is to use the Radon-Nikodým derivatives in order to obtain convenient expressions for the characteristic functions in terms of conditional expectations under the domestic spot martingale measure $\mathbb{P}$. The following lemma will allow us to achieve this goal.

Lemma 10. The following equality holds:

$$
\begin{aligned}
& \left.\frac{d \widehat{\mathbb{P}}_{T}}{d \mathbb{P}}\right|_{\mathscr{F}_{t}}=\exp \left(\int_{0}^{t} \sqrt{v_{u}} d W_{u}^{\mathrm{Q}}\right. \\
& -\int_{0}^{t} \sigma_{f} n_{f}(u, T) \sqrt{\widehat{r}_{u}} d W_{u}^{f} \\
& \left.-\frac{1}{2} \int_{0}^{t}\left(v_{u}+\sigma_{f}^{2} n_{f}^{2}(u, T) \widehat{r}_{u}\right) d u\right) .
\end{aligned}
$$

Proof. Straightforward computations show that

$$
\begin{aligned}
& \left.\frac{d \widehat{\mathbb{P}}_{T}}{d \mathbb{P}}\right|_{\mathscr{F}_{t}}=\left.\left.\frac{d \widehat{\mathbb{P}}_{T}}{d \mathbb{P}_{T}}\right|_{\mathscr{F}_{t}} \frac{d \mathbb{P}_{T}}{d \mathbb{P}}\right|_{\mathscr{F}_{t}} \\
& =\exp \left(\int_{0}^{t} \widetilde{\sigma}_{F}(u, T) \cdot d \widetilde{W}_{u}^{T}-\frac{1}{2} \int_{0}^{t}\left\|\widetilde{\sigma}_{F}(u, T)\right\|^{2} d u\right) \\
& \cdot \exp \left(-\int_{0}^{t} \sigma_{d} n_{d}(u, T) \sqrt{r_{u}} d W_{u}^{d}-\frac{1}{2}\right. \\
& \left.\cdot \int_{0}^{t} \sigma_{d}^{2} n_{d}^{2}(u, T) r_{u} d u\right)=\exp \left(\int_{0}^{t} \sqrt{v_{u}} d W_{u}^{\mathrm{Q}}\right. \\
& +\int_{0}^{t} \sigma_{d} n_{d}(u, T) \sqrt{r_{u}} d W_{u}^{T} \\
& \left.-\int_{0}^{t} \sigma_{f} n_{f}(u, T) \sqrt{\widehat{r}_{u}} d W_{u}^{f}\right) \exp \left(-\frac{1}{2}\right. \\
& \left.\cdot \int_{t}^{T}\left(v_{u}+\sigma_{d}^{2} n_{d}^{2}(u, T) r_{u}+\sigma_{f}^{2} n_{f}^{2}(u, T) \widehat{r}_{u}\right) d u\right)
\end{aligned}
$$

$$
\begin{aligned}
& \cdot \exp \left(-\int_{0}^{t} \sigma_{d} n_{d}(u, T) \sqrt{r_{u}} d W_{u}^{d}-\frac{1}{2}\right. \\
& \left.\cdot \int_{0}^{t} \sigma_{d}^{2} n_{d}^{2}(u, T) r_{u} d u\right) .
\end{aligned}
$$

Using (32), we now obtain

$$
\begin{aligned}
& \left.\frac{d \widehat{\mathbb{P}}_{T}}{d \mathbb{P}}\right|_{\mathscr{F}_{t}}=\exp \left(\int_{0}^{t} \sqrt{v_{u}} d W_{u}^{\mathrm{Q}}\right. \\
& -\int_{0}^{t} \sigma_{f} n_{f}(u, T) \sqrt{\widehat{r}_{u}} d W_{u}^{f} \\
& \left.-\frac{1}{2} \int_{0}^{t}\left(v_{u}+\sigma_{f}^{2} n_{f}^{2}(u, T) \widehat{r}_{u}\right) d u\right),
\end{aligned}
$$

which is the desired expression.

In view of the formula established in Lemma 10 and the abstract Bayes formula, to compute $f_{1}(\phi)=$ $f_{1}\left(\phi, t, Q_{t}, v_{t}, r_{t}, \widehat{r}_{t}\right)$, it suffices to focus on the following conditional expectation under $\mathbb{P}$ :

$$
\begin{gathered}
f_{1}(\phi)=\mathbb{E}_{t}^{\mathbb{P}}\left\{\operatorname { e x p } ( i \phi x _ { T } ) \operatorname { e x p } \left(\int_{t}^{T} \sqrt{v_{u}} d W_{u}^{\mathrm{Q}}\right.\right. \\
-\int_{t}^{T} \sigma_{f} n_{f}(u, T) \sqrt{\widehat{r}_{u}} d W_{u}^{f} \\
\left.\left.-\frac{1}{2} \int_{t}^{T}\left(v_{u}+\sigma_{f}^{2} n_{f}^{2}(u, T) \widehat{r}_{u}\right) d u\right)\right\} .
\end{gathered}
$$

Similarly, in view of formula (31), we obtain for $f_{2}(\phi)=$ $f_{2}\left(\phi, t, Q_{t}, v_{t}, r_{t}, \widehat{r}_{t}\right)$

$$
\begin{aligned}
& f_{2}(\phi)=\mathbb{E}_{t}^{\mathbb{P}}\left\{\exp \left(i \phi x_{T}\right)\right. \\
& \cdot \exp \left[-\int_{t}^{T} \sigma_{d} n_{d}(u, T) \sqrt{r_{u}} d W_{u}^{d}\right. \\
& \left.\left.-\frac{1}{2} \int_{t}^{T} \sigma_{d}^{2} n_{d}^{2}(u, T) r_{u} d u\right]\right\} .
\end{aligned}
$$

To proceed, we will need the following result, which is an immediate consequence of Lemma 7.

Corollary 11. Under Assumptions (A.1)-(A.4), the process $x_{t}=\ln F(t, T)$ admits the following representation under the domestic forward martingale measure $\mathbb{P}_{T}$ :

$$
\begin{aligned}
x_{T}= & x_{t}+\int_{t}^{T} \widetilde{\sigma}_{F}(u, T) \cdot d \widetilde{W}_{u}^{T}-\frac{1}{2} \int_{t}^{T}\left\|\widetilde{\sigma}_{F}(u, T)\right\|^{2} d u \\
& +J^{\mathrm{Q}}(t, T)-\lambda_{\mathrm{Q}} \mu_{\mathrm{Q}}(T-t),
\end{aligned}
$$


or, more explicitly,

$x_{T}$

$$
\begin{gathered}
=x_{t}+\int_{t}^{T} \sqrt{v_{u}} d W_{u}^{Q}+\int_{t}^{T} \sigma_{d} n_{d}(u, T) \sqrt{r_{u}} d W_{u}^{T} \\
-\int_{t}^{T} \sigma_{f} n_{f}(u, T) \sqrt{\widehat{r}_{u}} d W_{u}^{f}
\end{gathered}
$$

$$
-\frac{1}{2} \int_{t}^{T}\left(v_{u}+\sigma_{d}^{2} n_{d}^{2}(u, T) r_{u}+\sigma_{f}^{2} n_{f}^{2}(u, T) \widehat{r}_{u}\right) d u
$$$$
+\sum_{k=N_{t}^{\mathrm{Q}}+1}^{N_{T}^{\mathrm{Q}}} \ln \left(1+J_{k}^{\mathrm{Q}}\right)-\lambda_{\mathrm{Q}} \mu_{\mathrm{Q}}(T-t) .
$$

Using equality (50) and Corollary 11, we obtain

$$
\begin{aligned}
& f_{1}(\phi)=\mathbb{E}_{t}^{\mathbb{P}}\left\{\exp \left(i \phi x_{T}\right) \exp \left[\int_{t}^{T} \sqrt{v_{u}} d W_{u}^{\mathrm{Q}}-\int_{t}^{T} \sigma_{f} n_{f}(u, T) \sqrt{\widehat{r}_{u}} d W_{u}^{f}-\frac{1}{2} \int_{t}^{T}\left(v_{u}+\sigma_{f}^{2} n_{f}^{2}(u, T) \widehat{r}_{u}\right) d u\right]\right\} \\
& =\mathbb{E}_{t}^{\mathbb{P}}\left\{\exp \left[i \phi\left(x_{t}+\int_{t}^{T} \sqrt{v_{u}} d W_{u}^{\mathrm{Q}}+\int_{t}^{T} \sigma_{d} n_{d}(u, T) \sqrt{r_{u}} d W_{u}^{T}-\int_{t}^{T} \sigma_{f} n_{f}(u, T) \sqrt{\widehat{r}_{u}} d W_{u}^{f}\right)\right]\right. \\
& \cdot \exp \left[-\frac{i \phi}{2} \int_{t}^{T}\left(v_{u}+\sigma_{d}^{2} n_{d}^{2}(u, T) r_{u}+\sigma_{f}^{2} n_{f}^{2}(u, T) \widehat{r}_{u}\right) d u\right] \exp \left[\int_{t}^{T} \sqrt{v_{u}} d W_{u}^{\mathrm{Q}}-\int_{t}^{T} \sigma_{f} n_{f}(u, T) \sqrt{\widehat{r}_{u}} d W_{u}^{f}\right] \\
& \left.\cdot \exp \left[-\frac{1}{2} \int_{t}^{T}\left(v_{u}+\sigma_{f}^{2} n_{f}^{2}(u, T) \widehat{r}_{u}\right) d u\right]\right\} \exp \left[i \phi J^{\mathrm{Q}}(t, T)-i \phi \lambda_{\mathrm{Q}} \mu_{\mathrm{Q}}(T-t)\right] .
\end{aligned}
$$

For the sake of conciseness, we denote $\alpha=1+i \phi, \beta=i \phi$, and $c_{t}=\exp \left(i \phi x_{t}\right)$. After simplifications and rearrangement, the formula above becomes

$$
\begin{aligned}
& f_{1}(\phi)=c_{t} \mathbb{E}_{t}^{\mathbb{P}}\left\{\exp \left[\alpha\left(\int_{t}^{T} \sqrt{v_{u}} d W_{u}^{\mathrm{Q}}-\frac{1}{2} \int_{t}^{T} v_{u} d u\right)\right]\right. \\
& \cdot \exp \left[\beta\left(\int_{t}^{T} \sigma_{d} n_{d}(u, T) \sqrt{r_{u}} d W_{u}^{T}-\frac{1}{2} \int_{t}^{T} \sigma_{d}^{2} n_{d}^{2}(u, T) r_{u} d u\right)\right] \\
& \cdot \exp \left[-\alpha\left(\int_{t}^{T} \sigma_{f} n_{f}(u, T) \sqrt{\widehat{r}_{u}} d W_{u}^{f}+\frac{1}{2} \int_{t}^{T} \sigma_{f}^{2} n_{f}^{2}(u, T) \widehat{r}_{u} d u\right)\right] \\
& \left.\cdot \exp \left[\beta J^{\mathrm{Q}}(t, T)-\beta \lambda_{\mathrm{Q}} \mu_{\mathrm{Q}}(T-t)\right]\right\} .
\end{aligned}
$$

In view of Assumptions (A.1)-(A.6), we may use the following representation for the Brownian motion $W^{\mathrm{Q}}$ :

$$
W_{t}^{Q}=\rho W_{t}^{v}+\sqrt{1-\rho^{2}} W_{t}
$$

where $W=\left(W_{t}\right)_{t \in[0, T]}$ is a Brownian motion under $\mathbb{P}$ independent of the Brownian motions $W^{v}, W^{d}$, and $W^{f}$. Consequently, the conditional characteristic function $f_{1}(\phi)$ can be represented in the following way:

$$
\begin{aligned}
& f_{1}(\phi)=c_{t} \mathbb{E}_{t}^{\mathbb{P}}\left\{\operatorname { e x p } \left[\alpha \rho \int_{t}^{T} \sqrt{v_{u}} d W_{u}^{v}+\alpha \sqrt{1-\rho^{2}} \int_{t}^{T} \sqrt{v_{u}} d W_{u}\right.\right. \\
& \left.-\frac{\alpha}{2} \int_{t}^{T} v_{u} d u\right] \\
& \cdot \exp \left[\beta\left(\int_{t}^{T} \sigma_{d} n_{d}(u, T) \sqrt{r_{u}} d W_{u}^{T}-\frac{1}{2} \int_{t}^{T} \sigma_{d}^{2} n_{d}^{2}(u, T) r_{u} d u\right)\right] \\
& \cdot \exp \left[-\alpha\left(\int_{t}^{T} \sigma_{f} n_{f}(u, T) \sqrt{\widehat{r}_{u}} d W_{u}^{f}+\frac{1}{2} \int_{t}^{T} \sigma_{f}^{2} n_{f}^{2}(u, T) \widehat{r}_{u} d u\right)\right] \\
& \left.\cdot \exp \left[\beta J^{\mathrm{Q}}(t, T)-\beta \lambda_{\mathrm{Q}}(T-t) \mu_{\mathrm{Q}}\right]\right\} .
\end{aligned}
$$

Proof. The first asserted formula is an immediate consequence of (1). For the second, we recall that the function $n_{d}(t, T)$ is known to satisfy the following differential equation, for any fixed $T>0$ :

$$
\frac{\partial n_{d}(t, T)}{\partial t}-\frac{1}{2} \sigma_{d}^{2} n_{d}^{2}(t, T)-b_{d} n_{d}(t, T)+1=0
$$


with the terminal condition $n_{d}(T, T)=0$. Therefore, using the Itô formula and equality (32), we obtain

$$
\begin{aligned}
d\left(n_{d}(t, T) r_{t}\right)=r_{t} d n_{d}(t, T)+n_{d}(t, T) d r_{t} \\
=r_{t}\left(\frac{1}{2} \sigma_{d}^{2} n_{d}^{2}(t, T)+b_{d} n_{d}(t, T)-1\right) d t \\
\quad+n_{d}(t, T)\left(a_{d}-b_{d} r_{t}\right) d t+n_{d}(t, T) \sigma_{d} \sqrt{r_{t}} d W_{t}^{d} \\
=\frac{1}{2} \sigma_{d}^{2} n_{d}^{2}(t, T) r_{t} d t-r_{t} d t+n_{d}(t, T) a_{d} d t \\
\quad+n_{d}(t, T) \sigma_{d} \sqrt{r_{t}} d W_{t}^{d} \\
=-\frac{1}{2} \sigma_{d}^{2} n_{d}^{2}(t, T) r_{t} d t-r_{t} d t+n_{d}(t, T) a_{d} d t \\
\quad+n_{d}(t, T) \sigma_{d} \sqrt{r_{t}} d W_{t}^{T} .
\end{aligned}
$$

This yields the second asserted formula, upon integration between $t$ and $T$. The derivation of the last one is based on the same arguments and thus it is omitted.

4.2. Proof of Theorem 3. We split the proof of Theorem 3 into two steps in which we deal with $f_{1}(\phi)$ and $f_{2}(\phi)$.

Step 1. We will first compute $f_{1}(\phi)$. By combining (57) with the equalities derived in Lemma 12, we obtain the following representation for $f_{1}(\phi)$ :

$$
\begin{aligned}
& f_{1}(\phi)=c_{t} \mathbb{E}_{t}^{\mathbb{P}}\left\{\operatorname { e x p } \left[-\frac{\alpha \rho}{\sigma_{v}}\left(v_{t}+\theta \tau\right)\right.\right. \\
& +\left(\frac{\alpha \rho \kappa}{\sigma_{v}}-\frac{\alpha}{2}\right) \int_{t}^{T} v_{u} d u+\alpha \sqrt{1-\rho^{2}} \int_{t}^{T} \sqrt{v_{u}} d W_{u} \\
& \left.+\frac{\alpha \rho}{\sigma_{v}} v_{T}\right] \\
& \cdot \exp \left[-\beta\left(n_{d}(t, T) r_{t}+\int_{t}^{T} a_{d} n_{d}(u, T) d u\right)\right. \\
& \left.+\beta \int_{t}^{T} r_{u} d u\right] \\
& +\exp \left[\alpha\left(n_{f}(t, T) \widehat{r}_{t}+\int_{t}^{T} a_{f} n_{f}(u, T) d u\right)\right. \\
& \left.-\alpha \int_{t}^{T} \hat{r}_{u} d u\right] \exp \left[\beta J^{\mathrm{Q}}(t, T)-\beta \lambda_{\mathrm{Q}} \mu_{\mathrm{Q}}(T-t)\right. \\
& \left.\left.-\frac{\alpha \rho}{\sigma_{v}}\left(Z_{T}^{v}-Z_{t}^{v}\right)\right]\right\} .
\end{aligned}
$$

Recall the well-known property that if $\zeta$ has the standard normal distribution then $\mathbb{E}\left(e^{z \zeta}\right)=e^{z^{2} / 2}$ for any complex number $z \in \mathbb{C}$.
Consequently, by conditioning first on the sample path of the process $(v, r, \widehat{r})$ and using the independence of the processes $(v, r, \hat{r})$ and $W$ under $\mathbb{P}$ and Lemma 4 , we obtain

$$
\begin{aligned}
& f_{1}(\phi)=c_{t} \exp \left[\lambda_{\mathrm{Q}} \tau\left(\left(1+\mu_{\mathrm{Q}}\right)^{\beta} e^{-(1 / 2) \beta \gamma \sigma_{\mathrm{Q}}^{2}}-1\right)\right] \\
& \cdot \exp \left[-\left(\beta \lambda_{\mathrm{Q}} \mu_{\mathrm{Q}} \tau\right.\right. \\
& +\lambda_{v} \tau\left(\frac{\sigma_{v}\left(e^{-\left(\rho(1+i \phi) / \sigma_{v}\right) a}-e^{-\left(\rho(1+i \phi) / \sigma_{v}\right) b}\right)}{\rho(1+i \phi)(b-a)}+1\right) \\
& \left.\left.+\frac{\alpha \rho}{\sigma_{v}}\left(v_{t}+\theta \tau\right)\right)\right] \exp \left[-\beta\left(n_{d}(t, T) r_{t}\right.\right. \\
& \left.\left.+\int_{t}^{T} a_{d} n_{d}(u, T) d u\right)\right] \exp \left[\alpha \left(n_{f}(t, T) \widehat{r}_{t}\right.\right. \\
& \left.\left.+\int_{t}^{T} a_{f} n_{f}(u, T) d u\right)\right] \mathbb{E}_{t}^{\mathbb{P}}\left\{\operatorname { e x p } \left[\frac{\alpha \rho}{\sigma_{v}} v_{T}\right.\right. \\
& \left.+\left(\frac{\alpha^{2}\left(1-\rho^{2}\right)}{2}+\frac{\alpha \rho \kappa}{\sigma_{v}}-\frac{\alpha}{2}\right) \int_{t}^{T} v_{u} d u\right] \\
& \left.+\exp \left[\beta \int_{t}^{T} r_{u} d u-\alpha \int_{t}^{T} \widehat{r}_{u} d u\right]\right\}
\end{aligned}
$$

where we denote $\gamma=1-i \phi$. This in turn implies that the following equality holds:

$$
\begin{aligned}
& f_{1}(\phi)=c_{t} \exp \left[\lambda_{\mathrm{Q}} \tau\left(\left(1+\mu_{\mathrm{Q}}\right)^{\beta} e^{-(1 / 2) \beta \gamma \sigma_{\mathrm{Q}}^{2}}-1\right)\right] \\
& \cdot \exp \left[-\left(\beta \lambda_{\mathrm{Q}} \mu_{\mathrm{Q}} \tau\right.\right. \\
& +\lambda_{v} \tau\left(\frac{\sigma_{v}\left(e^{-\left(\rho(1+i \phi) / \sigma_{v}\right) a}-e^{-\left(\rho(1+i \phi) / \sigma_{v}\right) b}\right)}{\rho(1+i \phi)(b-a)}+1\right) \\
& \left.\left.+\frac{\alpha \rho}{\sigma_{v}}\left(v_{t}+\theta \tau\right)\right)\right] \exp \left[-\beta\left(n_{d}(t, T) r_{t}\right.\right. \\
& \left.\left.+\int_{t}^{T} a_{d} n_{d}(u, T) d u\right)\right] \exp \left[\alpha \left(n_{f}(t, T) \widehat{r}_{t}\right.\right. \\
& \left.\left.+\int_{t}^{T} a_{f} n_{f}(u, T) d u\right)\right] \mathbb{E}_{t}^{\mathbb{P}}\left\{\operatorname { e x p } \left[-s_{1} v_{T}\right.\right. \\
& \quad-s_{2} \int_{t}^{T} v_{u} d u-s_{3} r_{T}-s_{4} \int_{t}^{T} r_{u} d u-s_{5} \widehat{r}_{T} \\
& \left.\left.+s_{6} \int_{t}^{T} \widehat{r}_{u} d u\right]\right\},
\end{aligned}
$$

where the constants $s_{1}, s_{2}, s_{3}, s_{4}, s_{5}, s_{6}$ are given by (16). A direct application of Lemma 5 furnishes an explicit formula for $f_{1}(\phi)$, as reported in the statement of Theorem 3. 
Step 2. In order to compute the conditional characteristic function

$$
f_{2}(\phi)=f_{2}\left(\phi, t, Q_{t}, v_{t}, r_{t}, \widehat{r}_{t}\right)=\mathbb{E}_{t}^{\mathbb{P}_{T}}\left[\exp \left(i \phi x_{T}\right)\right],
$$

we proceed in an analogous manner as for $f_{1}(\phi)$. We first recall that (see (51))

$$
\begin{aligned}
& f_{2}(\phi)=\mathbb{E}_{t}^{\mathbb{P}}\left\{\exp \left(i \phi x_{T}\right)\right. \\
& \cdot \exp \left[-\int_{t}^{T} \sigma_{d} n_{d}(u, T) \sqrt{r_{u}} d W_{u}^{d}\right. \\
&\left.\left.-\frac{1}{2} \int_{t}^{T} \sigma_{d}^{2} n_{d}^{2}(u, T) r_{u} d u\right]\right\} .
\end{aligned}
$$

Therefore, using Corollary 11, we obtain

$$
\begin{aligned}
& f_{2}(\phi)=c_{t} \mathbb{E}_{t}^{\mathbb{P}}\left\{\exp \left[i \phi\left(\int_{t}^{T} \sqrt{v_{u}} d W_{u}^{\mathrm{Q}}+\int_{t}^{T} \sigma_{d} n_{d}(u, T) \sqrt{r_{u}} d W_{u}^{T}-\int_{t}^{T} \sigma_{f} n_{f}(u, T) \sqrt{\widehat{r}_{u}} d W_{u}^{f}\right)\right]\right. \\
& \cdot \exp \left[-i \phi\left(\frac{1}{2} \int_{t}^{T}\left(v_{u}+\sigma_{d}^{2} n_{d}^{2}(u, T) r_{u}+\sigma_{f}^{2} n_{f}^{2}(u, T) \widehat{r}_{u}\right) d u\right)\right] \\
& \left.\cdot \exp \left[-\int_{t}^{T} \sigma_{d} n_{d}(u, T) \sqrt{r_{u}} d W_{u}^{d}-\frac{1}{2} \int_{t}^{T} \sigma_{d}^{2} n_{d}^{2}(u, T) r_{u} d u\right] \exp \left[i \phi J^{\mathrm{Q}}(t, T)\right]\right\} .
\end{aligned}
$$

Consequently, using formulae (32) and (56) and Lemma 4, we obtain the following expression for $f_{2}(\phi)$ :

$$
\begin{aligned}
& f_{2}(\phi)=c_{t} \exp \left[\lambda_{\mathrm{Q}} \tau\left(\left(1+\mu_{\mathrm{Q}}\right)^{\beta} e^{-(1 / 2) \beta \gamma \sigma_{\mathrm{Q}}^{2}}-1\right)-\beta \lambda_{\mathrm{Q}} \mu_{\mathrm{Q}} \tau\right] \\
& \cdot \mathbb{E}_{t}^{\mathbb{P}}\left\{\exp \left[\beta\left(\rho \int_{t}^{T} \sqrt{v_{u}} d W_{u}^{v}+\sqrt{1-\rho^{2}} \int_{t}^{T} \sqrt{v_{u}} d W_{u}-\int_{t}^{T} \sigma_{f} n_{f}(u, T) \sqrt{\widehat{r}_{u}} d W_{u}^{f}\right)\right]\right. \\
&\left.\cdot \exp \left[-\beta\left(\frac{1}{2} \int_{t}^{T}\left(v_{u}+\sigma_{f}^{2} n_{f}^{2}(u, T) \widehat{r}_{u}\right) d u\right)\right] \exp \left[-\gamma\left(\int_{t}^{T} \sigma_{d} n_{d}(u, T) \sqrt{r_{u}} d W_{u}^{d}+\frac{1}{2} \int_{t}^{T} \sigma_{d}^{2} n_{d}^{2}(u, T) r_{u} d u\right)\right]\right\} .
\end{aligned}
$$

Similarly as in the case of $f_{1}(\phi)$, we condition on the sample path of the process $(v, r, \widehat{r})$ and we use the postulated independence of the processes $(v, r, \widehat{r})$ and $W$ under $\mathbb{P}$. By invoking also Lemma 4 , we obtain

$$
\begin{aligned}
& f_{2}(\phi)=c_{t} \exp \left[\lambda_{\mathrm{Q}} \tau\left(\left(1+\mu_{\mathrm{Q}}\right)^{\beta} e^{-(1 / 2) \beta \gamma \sigma_{Q}^{2}}-1\right)-\beta \lambda_{\mathrm{Q}} \mu_{\mathrm{Q}} \tau\right] \\
& \cdot \mathbb{E}_{t}^{\mathbb{P}}\left\{\exp \left[\beta \rho \int_{t}^{T} \sqrt{v_{u}} d W_{u}^{v}+\frac{\beta^{2}\left(1-\rho^{2}\right)-\beta}{2} \int_{t}^{T} v_{u} d u\right]\right. \\
& \cdot \exp \left[-\gamma\left(\int_{t}^{T} \sigma_{d} n_{d}(u, T) \sqrt{r_{u}} d W_{u}^{d}+\frac{1}{2} \int_{t}^{T} \sigma_{d}^{2} n_{d}^{2}(u, T) r_{u} d u\right)\right] \\
& \cdot \exp \left[-\beta\left(\int_{t}^{T} \sigma_{f} n_{f}(u, T) \sqrt{\widehat{r}_{u}} d W_{u}^{f}\right.\right. \\
& \left.\left.\left.+\frac{1}{2} \int_{t}^{T} \sigma_{f}^{2} n_{f}^{2}(u, T) \widehat{r}_{u} d u\right)\right]\right\} .
\end{aligned}
$$

Using Lemma 12, we conclude that

$$
\begin{gathered}
f_{2}(\phi)=c_{t} \exp \left[\lambda_{\mathrm{Q}} \tau\left(\left(1+\mu_{\mathrm{Q}}\right)^{\beta} e^{-(1 / 2) \beta \gamma \sigma_{\mathrm{Q}}^{2}}-1\right)\right] \\
\cdot \exp \left[-\left(\beta \lambda_{\mathrm{Q}} \mu_{\mathrm{Q}} \tau\right.\right.
\end{gathered}
$$

$$
\begin{aligned}
& +\lambda_{v} \tau\left[\frac{\sigma_{v}\left(e^{\left(\rho(i \phi) / \sigma_{v}\right) a}-e^{-\left(\rho(i \phi) / \sigma_{v}\right) b}\right)}{\rho(i \phi)(b-a)}+1\right] \\
& \left.\left.+\frac{\beta \rho}{\sigma_{v}}\left(v_{t}+\theta \tau\right)\right)\right] \exp \left[\gamma \left(n_{d}(t, T) r_{t}\right.\right. \\
& \left.\left.+\int_{t}^{T} a_{d} n_{d}(u, T) d u\right)\right] \exp \left[\beta \left(n_{f}(t, T) \widehat{r}_{t}\right.\right. \\
& \left.\left.+\int_{t}^{T} a_{f} n_{f}(u, T) d u\right)\right] \mathbb{E}_{t}^{\mathbb{P}}\left\{\operatorname { e x p } \left[-q_{1} v_{T}\right.\right. \\
& -q_{2} \int_{t}^{T} v_{u} d u-q_{3} r_{T}-q_{4} \int_{t}^{T} r_{u} d u-q_{5} \widehat{r}_{T} \\
& \left.\left.-q_{6} \int_{t}^{T} \widehat{r}_{u} d u\right]\right\},
\end{aligned}
$$

with the coefficients $q_{1}, q_{2}, q_{3}, q_{4}, q_{5}, q_{6}$ reported in formula (17). Another straightforward application of Lemma 5 yields the closed-form expression (14) for the conditional characteristic function $f_{2}(\phi)$. 
To complete the proof of Theorem 3, it suffices to combine formula (44) with the standard inversion formula (12) providing integral representations for the conditional probabilities:

$$
\begin{aligned}
& P_{1}\left(t, Q_{t}, v_{t}, r_{t}, \widehat{r}_{t}, K\right)=\widehat{\mathbb{P}}_{T}\left(x_{T}>\ln K \mid Q_{t}, v_{t}, r_{t}, \widehat{r}_{t}\right), \\
& P_{2}\left(t, Q_{t}, v_{t}, r_{t}, \widehat{r}_{t}, K\right)=\mathbb{P}_{T}\left(x_{T}>\ln K \mid Q_{t}, v_{t}, r_{t}, \widehat{r}_{t}\right) .
\end{aligned}
$$

This ends the derivation of the pricing formula for the foreign exchange call option. The price of the corresponding put option is readily available as well, due to the put-call parity relationship for FX options (see formula (72) in Section 5).

\section{Numerical Results}

The goal of the final section is to illustrate our approach by means of numerical examples in which we apply our FX market model, that is, the Heston/CIR jump-diffusion model, and we compare this approach with other related models that were proposed in Moretto et al. [30] and Ahlip and Rutkowski [1] to deal with the exchange rate derivatives.

Let us start by noting that the foreign exchange market differs from equity markets in that quotes for options are not made in terms of strikes. Indeed, the FX option prices are quoted in terms of the associated implied volatilities for a fixed forward delta $\Delta_{F}$ and a fixed time to expiry $\tau=T-t$. For more information about the market conventions, the interested reader is referred to, for instance, Moretto et al. [30] or Reiswich and Uwe [31].

For a quoted volatility $\sigma$, the corresponding strike price $K$ is obtained using the following conversion formula, which is based on the classic Garman-Kohlhagen lognormal model for the exchange rate:

$$
K=F(t, T) \exp \left(-\delta \sigma \sqrt{\tau} N^{-1}\left(\delta \Delta_{F}\right)+\frac{1}{2} \sigma^{2} \tau\right)
$$

where $N^{-1}$ is the inverse of the standard normal cumulative distribution function and the auxiliary parameter $\delta$ satisfies $\delta=1(\delta=-1$, resp.) for the call (put, resp.) option. Formula (71) makes it clear that market quotations prices based on the implied volatility for fixed deltas are in fact equivalent to quoting prices for fixed strikes. For more details, the interested reader is referred to Hakala and Wystup [32] or Reiswich and Uwe [31].

Another relevant feature is that currency derivatives are based on the notion of at-the-money forward (ATMF) rate, that is, the forward exchange rate $F(t, T)$ obtained by exploiting the interest rate parity implicit in (8). Recall that the universal put-call parity formula for plain-vanilla foreign exchange options reads

$$
C_{t}(T, K)-P_{t}(T, K)=Q_{t} B_{f}(t, T)-K B_{d}(t, T),
$$

where $C_{t}(T, K)$ and $P_{t}(T, K)$ are prices of currency call and put options, respectively. In particular, the prices of ATMF call and put options are equal in any arbitrage-free market model.
TABLE 1: Market volatility $\sigma_{\mathrm{MKT}}$ for USD/EUR derivative exchange rate on June 13, 2005 (original source of data: Banca Caboto S.p.A., Gruppo Intesa, Milano).

\begin{tabular}{lccccccc}
\hline Delta & $-10 \%$ & $-15 \%$ & $-25 \%$ & $\begin{array}{c}\text { ATMF } \\
(50 \%)\end{array}$ & $25 \%$ & $15 \%$ & $10 \%$ \\
\hline $1 \mathrm{M}$ & $10.36 \%$ & $10.09 \%$ & $9.73 \%$ & $9.30 \%$ & $9.15 \%$ & $9.18 \%$ & $9.25 \%$ \\
$2 \mathrm{M}$ & $10.28 \%$ & $10.01 \%$ & $9.65 \%$ & $9.25 \%$ & $9.15 \%$ & $9.22 \%$ & $9.31 \%$ \\
$3 \mathrm{M}$ & $10.22 \%$ & $9.95 \%$ & $9.62 \%$ & $9.25 \%$ & $9.19 \%$ & $9.28 \%$ & $9.39 \%$ \\
$6 \mathrm{M}$ & $10.23 \%$ & $9.95 \%$ & $9.64 \%$ & $9.35 \%$ & $9.39 \%$ & $9.55 \%$ & $9.74 \%$ \\
$9 \mathrm{M}$ & $10.22 \%$ & $9.96 \%$ & $9.96 \%$ & $9.40 \%$ & $9.49 \%$ & $9.68 \%$ & $9.88 \%$ \\
$1 \mathrm{Y}$ & $10.24 \%$ & $9.98 \%$ & $9.69 \%$ & $9.45 \%$ & $9.56 \%$ & $9.77 \%$ & $9.99 \%$ \\
$2 \mathrm{Y}$ & $10.28 \%$ & $10.02 \%$ & $9.74 \%$ & $9.55 \%$ & $9.72 \%$ & $9.98 \%$ & $10.24 \%$ \\
\hline
\end{tabular}

TABLe 2: Market strike prices for USD/EUR derivative exchange rate on June 13, 2005 (original source of data: Banca Caboto S.p.A., Gruppo Intesa, Milano).

\begin{tabular}{lccccccc}
\hline Strike & $-10 \%$ & $-15 \%$ & $-25 \%$ & $\begin{array}{r}\text { ATMF } \\
(50 \%)\end{array}$ & $25 \%$ & $15 \%$ & $10 \%$ \\
\hline $1 \mathrm{M}$ & 1.1651 & 1.1745 & 1.1877 & 1.2101 & 1.2317 & 1.2435 & 1.2519 \\
$2 \mathrm{M}$ & 1.1496 & 1.1626 & 1.1807 & 1.2116 & 1.2421 & 1.2591 & 1.2712 \\
$3 \mathrm{M}$ & 1.1370 & 1.1529 & 1.1752 & 1.2134 & 1.2518 & 1.2735 & 1.2891 \\
$6 \mathrm{M}$ & 1.1129 & 1.1350 & 1.1660 & 1.2189 & 1.2753 & 1.3081 & 1.3324 \\
$9 \mathrm{M}$ & 1.0968 & 1.1233 & 1.1609 & 1.2246 & 1.2951 & 1.3369 & 1.3680 \\
$1 \mathrm{Y}$ & 1.0843 & 1.1147 & 1.1579 & 1.2307 & 1.3140 & 1.3638 & 1.4013 \\
$2 \mathrm{Y}$ & 1.0561 & 1.0984 & 1.1596 & 1.2562 & 1.3826 & 1.4606 & 1.5205 \\
\hline
\end{tabular}

TABLE 3: Market domestic (USD) and foreign (EUR) interest rates on June 13, 2005 (original source of data: Banca Caboto S.p.A., Gruppo Intesa, Milano).

\begin{tabular}{lcc}
\hline Rates & $r_{d}$ & $r_{f}$ \\
\hline $1 \mathrm{M}$ & $3.14 \%$ & $2.09 \%$ \\
$2 \mathrm{M}$ & $3.22 \%$ & $2.09 \%$ \\
$3 \mathrm{M}$ & $3.32 \%$ & $2.10 \%$ \\
$6 \mathrm{M}$ & $3.50 \%$ & $2.09 \%$ \\
$9 \mathrm{M}$ & $3.60 \%$ & $2.09 \%$ \\
$1 \mathrm{Y}$ & $3.68 \%$ & $2.09 \%$ \\
$2 \mathrm{Y}$ & $4.02 \%$ & $2.19 \%$ \\
\hline
\end{tabular}

5.1. Market Data. In the numerical results presented in Tables 1, 2, and 3, we make use (with the kind permission of the authors) of the data for the USD/EUR exchange rate derivatives and interest rates from the paper by Moretto et al. [30] (see page 469 therein).

5.2. Comparison of Model Prices. The dynamics of the exchange rate and volatility, as given by (1), involve the parameters: $\lambda_{\mathrm{Q}}, \mu_{\mathrm{Q}}, \sigma_{\mathrm{Q}}, \lambda_{v}, \kappa, \theta$, and $\sigma_{v}$. In addition, there are three parameters for each of the interest rates. In our numerical examples, the values of parameters $\kappa, \theta$, and $\sigma_{v}$ are borrowed from Moretto et al. [30], who proposed an extension of the Heston model for the exchange rate under the 
TABLE 4: Values of parameters of the HCIR/LN/LU model (1).

\begin{tabular}{ccccccccc}
\hline$a$ & $b$ & $\theta$ & $\kappa$ & $\lambda_{\mathrm{Q}}$ & $\mu_{\mathrm{Q}}$ & $\lambda_{v}$ & $\rho$ & $\sigma_{\mathrm{Q}}$ \\
\hline 0.1 & 0.2 & 0.02606 & 0.091 & 0.1000 & 0.00258 & 0.1000 & 0.9786 & 0.0644 \\
\hline
\end{tabular}

TABLE 5: Prices of ATM USD/EUR European exchange rate call options using data of June 13, 2005.

\begin{tabular}{lccc}
\hline Maturity & Heston price & HCIR price & HCIR-LN-LU price \\
\hline $1 \mathrm{M}$ & 0.0128496 & 0.0128912 & 0.0129512 \\
$2 \mathrm{M}$ & 0.0190734 & 0.0192465 & 0.0233037 \\
$3 \mathrm{M}$ & 0.0245511 & 0.0249469 & 0.0322193 \\
$6 \mathrm{M}$ & 0.0390888 & 0.0407209 & 0.0585503 \\
$9 \mathrm{M}$ & 0.0526127 & 0.0556335 & 0.0816888 \\
$12 \mathrm{M}$ & 0.0656178 & 0.0699566 & 0.102082 \\
\hline
\end{tabular}

TABle 6: Prices of 25\% USD/EUR European exchange rate call options using data of June 13, 2005.

\begin{tabular}{lccc}
\hline Maturity & Heston price & HCIR price & HCIR-LN-LU price \\
\hline $1 \mathrm{M}$ & 0.0005469 & 0.0054274 & 0.0062094 \\
$2 \mathrm{M}$ & 0.0088177 & 0.0089194 & 0.0140153 \\
$3 \mathrm{M}$ & 0.0116882 & 0.0119536 & 0.0224628 \\
$6 \mathrm{M}$ & 0.0205735 & 0.0216388 & 0.0457036 \\
$9 \mathrm{M}$ & 0.0297421 & 0.0323061 & 0.0663260 \\
$12 \mathrm{M}$ & 0.0389762 & 0.0437765 & 0.0849682 \\
\hline
\end{tabular}

assumption of constant interest rates, as represented by the market yield curve. It should be acknowledged that the choice of interest rate parameters in our model is rather artificial and it was made for illustrative purposes only. We used the following values of parameters for the Heston/CIR (HCIR) model and the Heston/CIR/Log Normal/Log Uniform JumpDiffusion (HCIR-LN-LU) model: $a_{d}=0.0332 ; b_{d}=0.03$; $a_{f}=0.021 ; b_{f}=0.024 ; \sigma_{d}=0.25 ; \sigma_{f}=0.24 ; Q_{0}=1.2087 ;$ and $\rho=0.9786$. For each maturity date, the initial value $v_{0}=0.0078$. The parameters given in Table 4 were taken from D'Ippoliti et al. [10] and were used for illustrative purposes only. The Heston model, the HCIR model examined in Ahlip and Rutkowski [1], and the HCIR-LN-LU model put forward in this paper were compared. Although the numerical results presented here are only preliminary, they nevertheless make it clear that jumps in exchange rate and volatility dynamics and the uncertain character of interest rates affect the valuation of foreign exchange derivatives.

In Table 5, we report prices of ATM calls for expiries ranging from one month to one year. We use here the ATM volatilities for different maturities given in Table 1, the corresponding ATM strike prices from Table 2, and the interest rates from Table 3. As one can see, the prices obtained using our model (HCIR-LN-LU) are higher than the prices for HCIR model and substantially higher than the prices obtained for the Heston model. In Table 6, we report $25 \%$ USD/EUR currency call option prices computed in the Heston model (refer to Figures 1 and 4), the HCIR model, and the present model using data of June 13, 2005. In the next three examples (see Tables 7, 8, and 9) we consider prices for

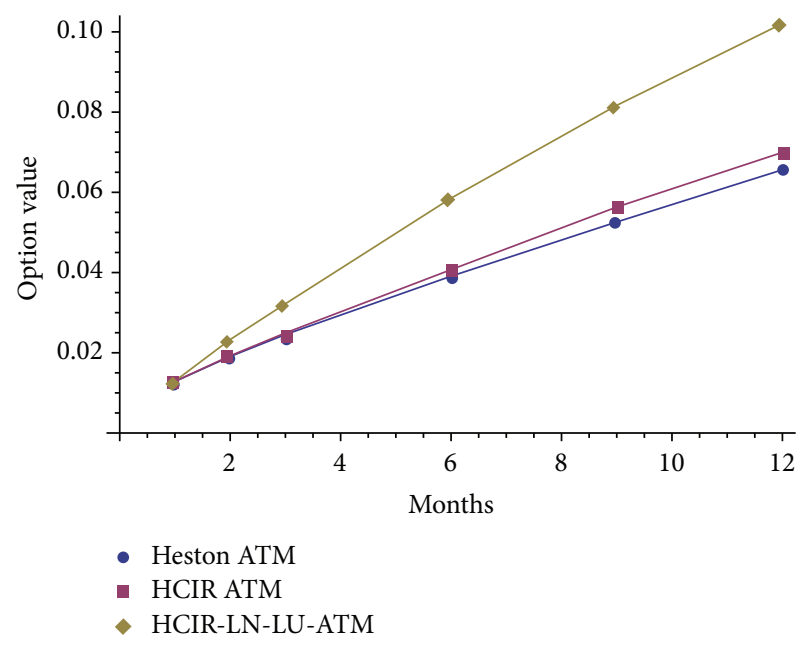

FIgURE 1: Graphs for ATM options prices given in Table 5.

TAble 7: Prices for ATM USD/EUR European exchange rate call options using data of June 13, 2005. Values in brackets are strike levels obtained using formula (71).

\begin{tabular}{lccc}
\hline Maturity & Heston price & HCIR price & HCIR-LN-LU price \\
\hline \multirow{2}{*}{$1 \mathrm{M}$} & 0.0128496 & 0.0128546 & 0.0128697 \\
& $(1.21019)$ & $(1.21028)$ & $(1.21028)$ \\
\hline \multirow{2}{*}{$2 \mathrm{M}$} & 0.0190734 & 0.0190999 & 0.0222887 \\
& $(1.21184)$ & $(1.21217)$ & $(1.21217)$ \\
\hline \multirow{2}{*}{$3 \mathrm{M}$} & 0.024422 & 0.02449323 & 0.0319434 \\
& $(1.21369)$ & $(1.21428)$ & $(1.21428)$ \\
\hline \multirow{2}{*}{$6 \mathrm{M}$} & 0.0386608 & 0.0390158 & 0.0573285 \\
& $(1.21992)$ & $(1.22289)$ & $(1.22289)$ \\
\hline \multirow{2}{*}{$9 \mathrm{M}$} & 0.0518264 & 0.0527189 & 0.0790228 \\
& $(1.22652)$ & $(1.23329)$ & $(1.23329)$ \\
\hline \multirow{2}{*}{$12 \mathrm{M}$} & 0.0644786 & 0.0681417 & 0.0988909 \\
& $(1.23356)$ & $(1.24071)$ & $(1.24071)$ \\
\hline
\end{tabular}

TABLE 8: Prices for 25\% USD/EUR European exchange rate call options using data of June 13, 2005. Values in brackets are strike levels obtained using formula (71).

\begin{tabular}{lccc}
\hline Maturity & Heston price & HCIR price & HCIR-LN-LU price \\
\hline \multirow{2}{*}{$1 \mathrm{M}$} & 0.0054139 & 0.0054181 & 0.0058999 \\
& $(1.23193)$ & $(1.23201)$ & $(1.23201)$ \\
\hline \multirow{2}{*}{$2 \mathrm{M}$} & 0.0086633 & 0.0086839 & 0.0138257 \\
& $(1.24274)$ & $(1.24308)$ & $(1.24308)$ \\
\hline \multirow{2}{*}{$3 \mathrm{M}$} & 0.0116882 & 0.0117434 & 0.0232394 \\
& $(1.25188)$ & $(1.25267)$ & $(1.25267)$ \\
\hline \multirow{2}{*}{$6 \mathrm{M}$} & 0.0204432 & 0.0207368 & 0.0480355 \\
& $(1.27581)$ & $(1.27892)$ & $(1.27892)$ \\
\hline \multirow{2}{*}{$9 \mathrm{M}$} & 0.0293928 & 0.0301153 & 0.0706473 \\
& $(1.29652)$ & $(1.30367)$ & $(1.30367)$ \\
\hline \multirow{2}{*}{$12 \mathrm{M}$} & 0.0385139 & 0.0399506 & 0.0909937 \\
& $(1.31587)$ & $(1.32884)$ & $(1.32884)$ \\
\hline
\end{tabular}


TABLE 9: Prices for 15\% USD/EUR European exchange rate call options using data of June 13, 2005. Values in brackets are strike levels obtained using formula (71).

\begin{tabular}{lccc}
\hline Maturity & Heston price & HCIR price & HCIR-LN-LU price \\
\hline \multirow{2}{*}{$1 \mathrm{M}$} & 0.0031477 & 0.0031503 & 0.0032142 \\
& $(1.24388)$ & $(1.24397)$ & $(1.24397)$ \\
\hline \multirow{2}{*}{$2 \mathrm{M}$} & 0.0005302 & 0.0053178 & 0.0120455 \\
& $(1.26005)$ & $(1.27429)$ & $(1.27429)$ \\
\hline \multirow{2}{*}{$3 \mathrm{M}$} & 0.0074126 & 0.0074553 & 0.0208664 \\
& $(1.27349)$ & $(1.27429)$ & $(1.27429)$ \\
\hline \multirow{2}{*}{$6 \mathrm{M}$} & 0.0138051 & 0.0140366 & 0.0433107 \\
& $(1.30848)$ & $(1.311672$ & $(1.31167)$ \\
\hline \multirow{2}{*}{$9 \mathrm{M}$} & 0.0207329 & 0.0213553 & 0.0616101 \\
& $(1.33813)$ & $(1.34551)$ & $(1.34551)$ \\
\hline \multirow{2}{*}{$1 \mathrm{Y}$} & 0.0280719 & 0.0293209 & 0.0437222 \\
& $(1.36544)$ & $(1.33788)$ & $(1.33788)$ \\
\hline
\end{tabular}

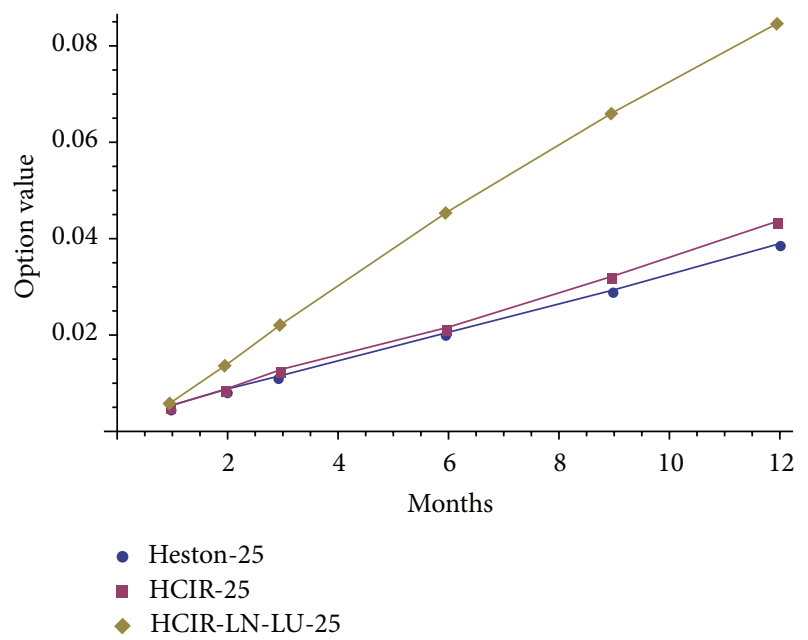

FiguRE 2: Graphs for 25\% options prices given in Table 6.

ATM and $25 \%$ and $15 \%$ volatilities (refer to Figures 3, 2, and 5), respectively. For each maturity, the corresponding strike level was obtained using (71). Prices for ATM, 25\%, and 15\% USD/EUR currency call options are computed in the Heston, HCIR, and HCIR-LN-LU models using data of June 13, 2005, and parameter values given in Table 4 .

\section{Conflict of Interests}

The authors declare that there is no conflict of interests regarding the publication of this paper.

\section{Acknowledgments}

The authors are grateful to Enrico Moretto for consenting to use data reported in [30] and Uwe Wystup who kindly advised them in regards to numerical examples presented in Section 5. They also thank Marek Rutkowski, Scott Joslin,

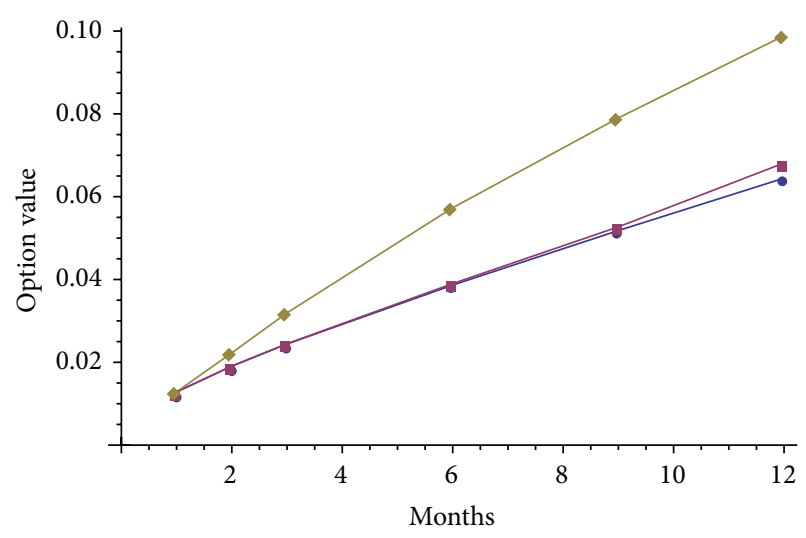

- Heston ATM

- hCIR ATM

- HCIR-LN-LU-ATM

FIgURE 3: Graphs for options prices given in Table 7.

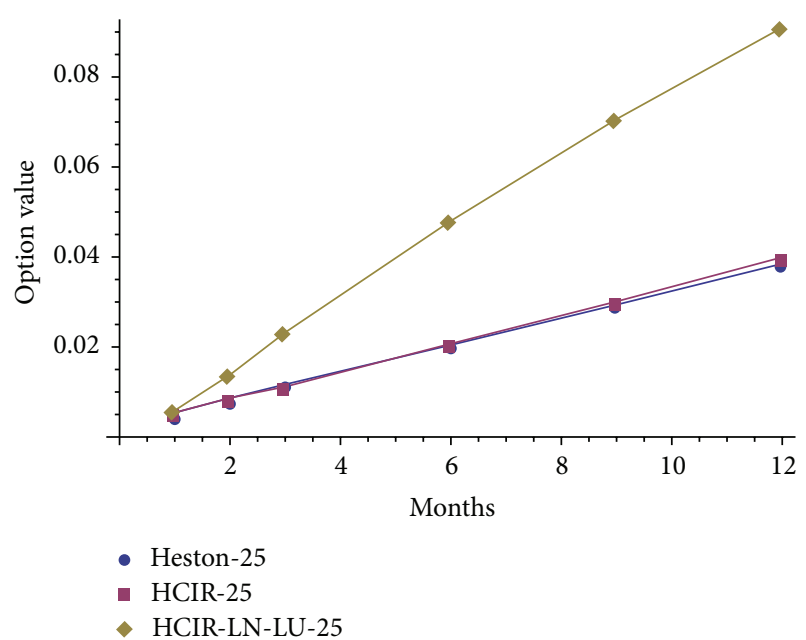

Figure 4: Graphs for options prices given in Table 8.

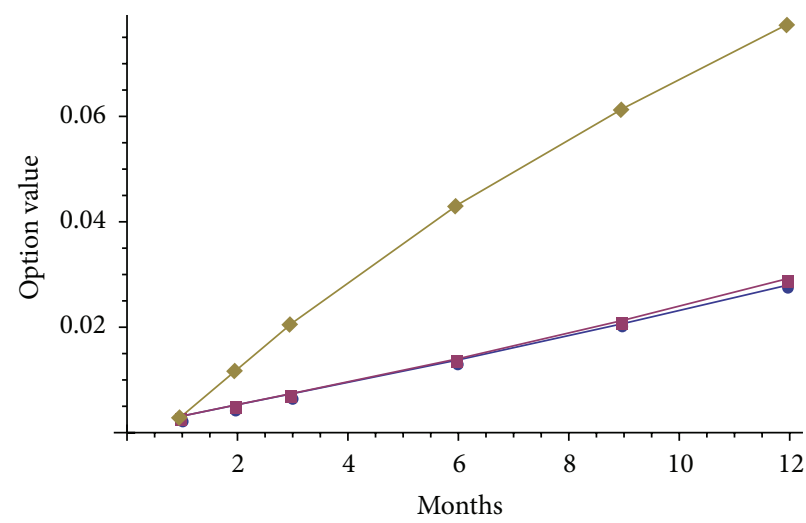

- Heston ATM

- hCIR ATM

- HCIR-LN-LU-ATM

FIGURE 5: Graph for option 15\% prices given in Table 9. 
Paul Glasserman, and participants at the IMS-FPS-2014 Workshop, July 2-6, 2014, UTS, Sydney, for valuable comments on an earlier version of the paper. All remaining errors are theirs.

\section{References}

[1] R. Ahlip and M. Rutkowski, "Pricing of foreign exchange options under the Heston stochastic volatility model and CIR interest rates," Quantitative Finance, vol. 13, no. 6, pp. 955-966, 2013.

[2] J. C. Cox, J. E. Ingersoll, and S. A. Ross, "A theory of the term structure of interest rates," Econometrica, vol. 53, no. 2, pp. 385407, 1985.

[3] S. L. Heston, "A closed-form solution for options with stochastic volatility with applications to bond and currency options," Review of Financial Studies, vol. 6, no. 2, pp. 327-343, 1993.

[4] G. Bakshi, C. Cao, and Z. Chen, "Empirical performance of alternative option pricing models," Journal of Finance, vol. 52, no. 5, pp. 2003-2049, 1997.

[5] D. S. Bates, "Jumps and stochastic volatility: exchange rate processes implicit in Deutsche Mark options," Review of Financial Studies, vol. 9, no. 1, pp. 69-107, 1996.

[6] D. Duffie, J. Pan, and K. Singleton, "Transform analysis and asset pricing for affine jump-diffusions," Econometrica, vol. 68, no. 6, pp. 1343-1376, 2000.

[7] T. Andersen and J. Andreasen, "Jump-diffusion processes: volatility smile fitting and numerical methods," The Journal of Financial Economics, vol. 4, pp. 231-262, 2000.

[8] S. Alizadeh, M. W. Brandt, and F. X. Diebold, "Range-based estimation of stochastic volatility models," The Journal of Finance, vol. 57, no. 3, pp. 1047-1091, 2002.

[9] B. Eraker, M. Johannes, and N. Polson, "The impacts of jumps in volatility and returns," Journal of Finance, vol. 58, no. 3, pp. 1269-1300, 2003.

[10] F. D’Ippoliti, E. Moretto, S. Pasquali, and B. Trivellato, "Exact pricing with stochastic volatility and jumps," International Journal of Theoretical and Applied Finance, vol. 13, no. 6, pp. 901929, 2010.

[11] G. Yan and F. B. Hanson, "Option pricing for stochastic volatility jump diffusion model with log-uniform jump-amplitudes," in Proceedings of the American Control Conference, pp. 1-7, September 2006.

[12] A. van Haastrecht, R. Lord, A. Pelsser, and D. Schrager, "Pricing long-dated insurance contracts with stochastic interest rates and stochastic volatility," Insurance: Mathematics and Economics, vol. 45, no. 3, pp. 436-448, 2009.

[13] R. Schobel and J. Zhu, "Stochastic volatility with an ornsteinuhlenbeck process: an extension," Review of Finance, vol. 3, no. 1, pp. 23-46, 1999.

[14] O. Vasicek, "An equilibrium characterization of the term structure," Journal of Financial Economics, vol. 5, no. 2, pp. 177-188, 1977.

[15] A. van Haastrecht and A. Pelsser, "Generic pricing of FX, inflation and stock options under stochastic interest rates and stochastic volatility," Quantitative Finance, vol. 11, no. 5, pp. 665691, 2011.

[16] L. A. Grzelak and C. W. Oosterlee, "On the Heston model with stochastic interest rates," SIAM Journal on Financial Mathematics, vol. 2, no. 1, pp. 255-286, 2011.
[17] L. A. Grzelak and C. W. Oosterlee, "On cross-currency models with stochastic volatility and correlated interest rates," Applied Mathematical Finance, vol. 19, no. 1, pp. 1-35, 2012.

[18] L. A. Grzelak, C. W. Oosterlee, and S. van Weeren, "Extension of stochastic volatility equity models with the Hull-White interest rate process," Quantitative Finance, vol. 12, no. 1, pp. 89-105, 2012.

[19] B. Wong and C. C. Heyde, "On the martingale property of stochastic exponentials," Journal of Applied Probability, vol. 41, no. 3, pp. 654-664, 2004.

[20] M. Musiela and M. Rutkowski, Martingale Methods in Financial Modelling, vol. 36 of Stochastic Modelling and Applied Probability, Springer, New York, NY, USA, 2nd edition, 2005.

[21] P. Carr and D. Madan, "Option valuation using the fast fourier transform," Journal of Computational Finance, vol. 2, pp. 61-73, 1999.

[22] P. Carr and D. Madan, "Saddlepoint methods for option pricing," Journal of Computational Finance, vol. 13, no. 1, pp. 4961, 2009.

[23] R. Lord and C. Kahl, "Optimal fourier inversion in semianalytical option pricing," Journal of Computational Finance, vol. 10, pp. 1-30, 2007.

[24] R. Lord and C. Kahl, "Complex logarithms in Heston-like models," Mathematical Finance, vol. 20, no. 4, pp. 671-694, 2010.

[25] C. Bernard, Z. Cui, and D. McLeish, "Nearly exact option price simulation using characteristic functions," International Journal of Theoretical and Applied Finance, vol. 15, no. 7, Article ID 1250047, 29 pages, 2012.

[26] S. Levendorskii, "Efficient pricing and reliable calibration in the Heston model," International Journal of Theoretical and Applied Finance, vol. 15, Article ID 1250050, 44 pages, 2012.

[27] M. Jeanblanc, M. Yor, and M. Chesney, Mathematical methods for financial markets, Springer Finance, Springer, London, UK, 2009.

[28] R. Ahlip and M. Rutkowski, "Forward start options under stochastic volatility and stochastic interest rates," International Journal of Theoretical and Applied Finance, vol. 12, no. 2, pp. 209-225, 2009.

[29] D. Brigo and A. Alfonsi, "Credit default swap calibration and derivatives pricing with the SSRD stochastic intensity model," Finance and Stochastics, vol. 9, no. 1, pp. 29-42, 2005.

[30] E. Moretto, S. Pasquali, and B. Trivellato, "Derivative evaluation using recombining trees under stochastic volatility," Advances and Applications in Statistical Sciences, vol. 1, no. 2, pp. 453-480, 2010.

[31] D. Reiswich and W. Uwe, "FX volatility smile construction," Wilmott, vol. 2012, no. 60, pp. 58-69, 2012.

[32] J. Hakala and U. Wystup, Foreign Exchange Risk: Models, Instruments and Strategies, Risk Books, London, UK, 2002. 


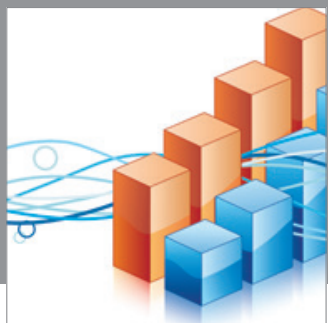

Advances in

Operations Research

mansans

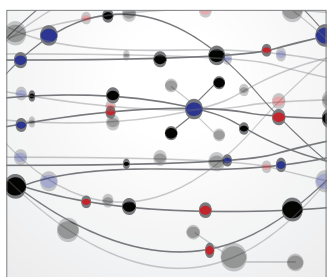

The Scientific World Journal
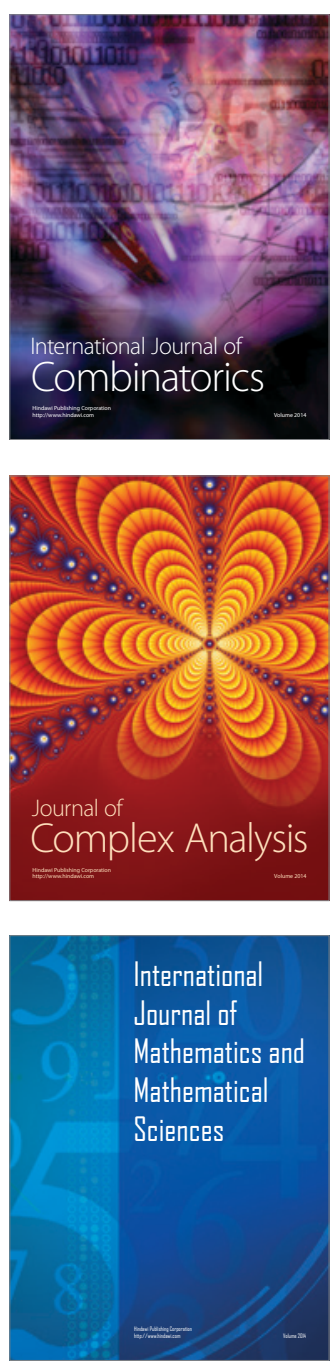
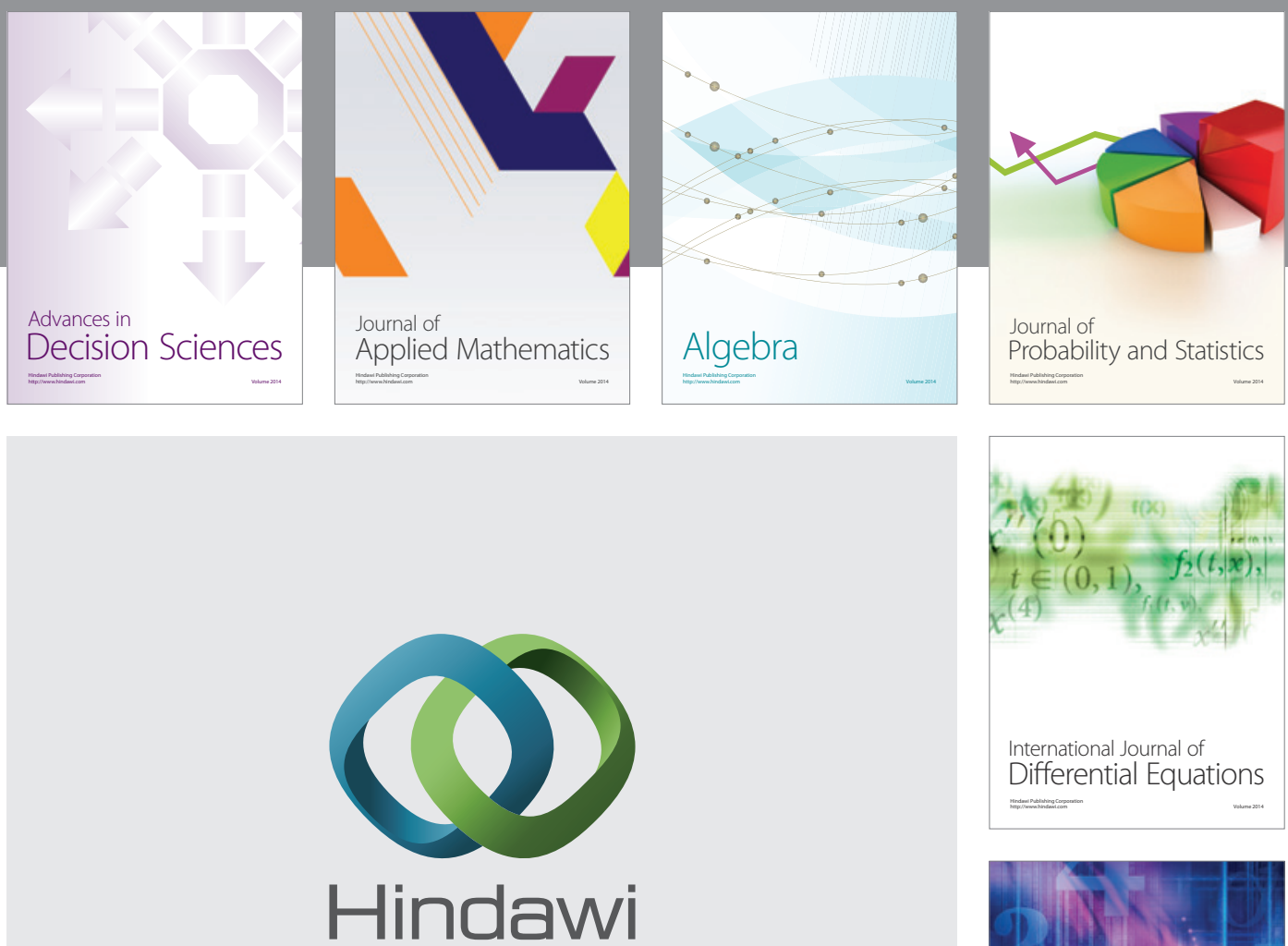

Submit your manuscripts at http://www.hindawi.com
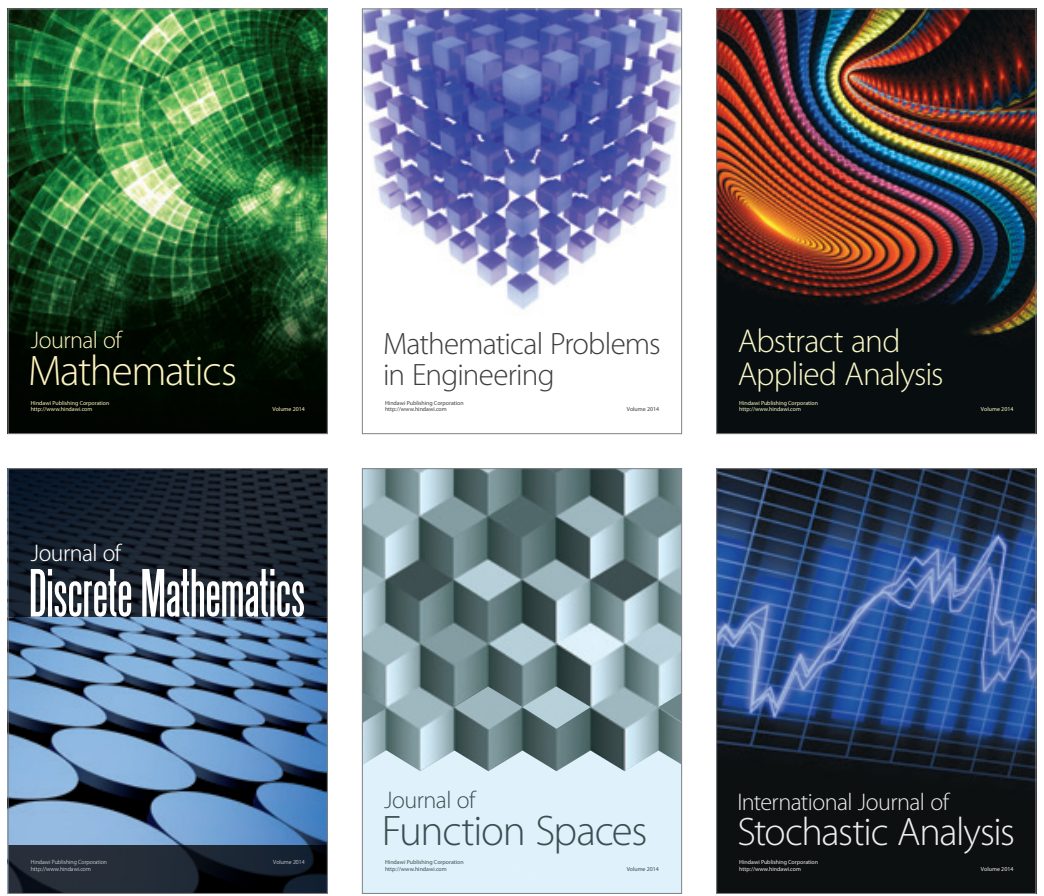

Journal of

Function Spaces

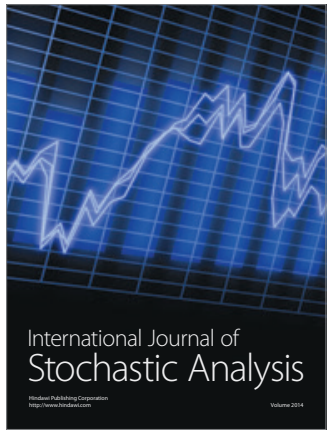

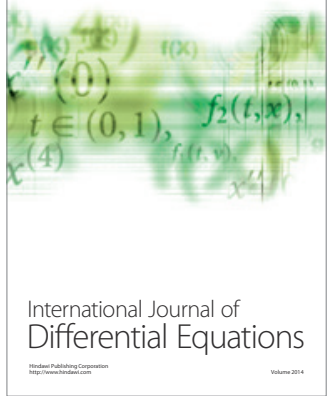
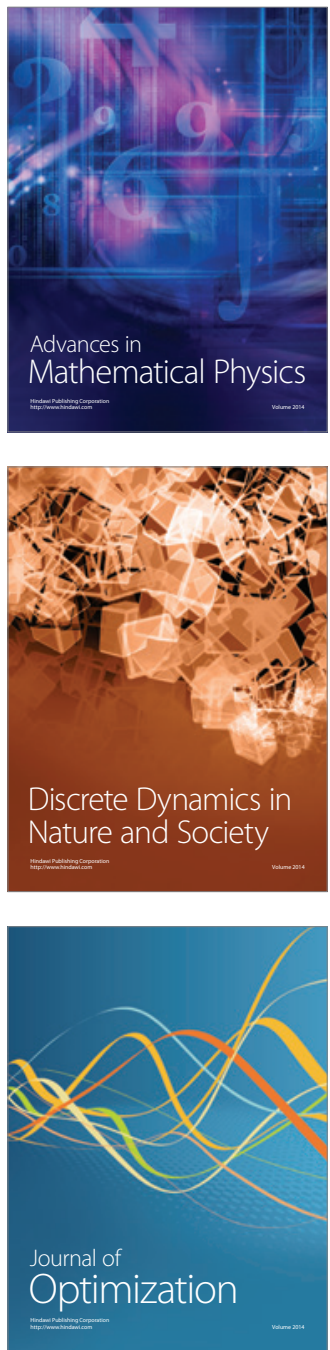